\title{
Smooth Transitions for a Turning Dubins Vehicle
}

\author{
Heejun Choi* and Ella M. Atkins ${ }^{\dagger}$ \\ University of Michigan, Ann Arbor, MI 48105
}

\begin{abstract}
Emergencies in which an aircraft cannot maintain straight flight can result from a variety of conditions such as structural damage or actuator failures. A Turning Dubins Vehicle (TDV) is defined as an analytical flight path planner for situations in which straight flight is not possible. Solutions are constructed as a sequence of alternate turning arcs that follow a reference circle and include piecewise linear transitions between turning radii. A comprehensive Turning Dubins Vehicle (TDV) solver is presented to handle the spectrum of relative distances and headings between aircraft initial state and a landing runway approach end. This solver generates the minimum number of turning sequences for the TDV, thus providing a minimum length landing flight plan. Example solutions are used to illustrate the TDV solver.
\end{abstract}

\section{Nomenclature}

$\sigma \quad$ Curve (Lateral Plane Landing Path) for the Turning Dubins Vehicle (TDV)

$\mathcal{O}$ Circular curve for the TDV

a Circular arc curve for the TDV

$b \quad$ Product of circular arc curves

$\Sigma \quad$ Set of possible curves for the TDV

$\Sigma_{c} \quad$ Set of circular curves for the TDV

$\mathcal{A} \quad$ Set of circular arc curves for the TDV

$\mathcal{B} \quad$ Set of possible sequences of two different turning radii for the TDV

$\mathcal{C}$ Natural representation of $\sigma$ with respect to a center $c_{1}$ of the first circular curve for the TDV

$\mathcal{R}_{\mathcal{O}} \quad$ Set of possible reference circles having $\overline{c_{1} c_{f}}$ as a chord

$s \quad$ Length of the arc segment of the $\sigma$

$\vec{V} \quad$ Velocity vector

$\vec{T} \quad$ Unit tangent with respect to $c_{1}$

$\vec{k} \quad$ Curvature vector with respect to $c_{1}$

$\vec{n} \quad$ Principal normal unit vector with respect to $c_{1}$

$\vec{b} \quad$ Unit binormal vector with respect to $c_{1}$

$r \quad$ Radius of circular curve

$\delta \quad$ Central angle of the reference arc

$\psi \quad$ Angle between unit tangent $\vec{T}$ and unit vector $\hat{i}$ in the $x y z$ system

$n \quad$ Number of arc sequences in $\mathcal{B}$

$n_{m} \quad$ Minimum number of arc sequences in $\mathcal{B}$

$\lambda \quad$ Distance of the points of a straight line from a known point

Subscript

$r \quad$ Reference circle

1 First circular curve of two different radius circular curves

2 Second circular curve of two different radius circular curves

$m \quad$ Minimum radius turning circle

$M \quad$ Maximum radius turning circle

$\mathrm{T} \quad$ Transition for the TDV

*Research Assistant, Aerospace Engineering, University of Michigan, Ann Arbor, MI 48105

${ }^{\dagger}$ Associate Professor, Aerospace Engineering, University of Michigan, Ann Arbor, MI 48105, Associate Fellow 


\section{Introduction}

Modern flight management systems (FMS) can generate optimal flight plans over a variety of conditions. When damage or failures occur, such as structural damage, loss of thrust, or a control surface jam, the flight crew must manually adapt the flight plans to execute an emergency landing, ideally at a nearby airport. Pilot workload is dramatically increased in such situations, particularly given the split in attention between attempting to understand the reduced performance properties as well as manually devise a guidance strategy (and flight plan) to safely land the aircraft. To assist the flight crew with adaptive guidance as well as flight control given degraded performance, researchers have begun to develop emergency real-time landing trajectory generation automation aids, particularly given that existing flight plans may not be feasible. ${ }^{1-4}$ In our past work, an adaptive flight planning capability is defined to automatically rank and select a nearby landing runway then construct a trajectory to that runway under the assumption that either a Dubins path solution could be found or that sufficient flight planning time exists to identify a landing trajectory via an optimal search over sequences of feasible trim states. The Dubins solver requires straight flight segments connected by turns, while the search-based algorithm might not guarantee a solution is identified within an acceptable time interval. We have previously defined a Turning Dubins Vehicle (TDV) trajectory planner ${ }^{5}$ to efficiently guide a damaged aircraft to a chosen landing runway. With the goal of providing a computationally-efficient and provably-correct analogue to the Dubins solver, a sequence of alternating extreme turning arcs are generated that follow maximum and minimum radii paths, respectively, to enable a feasible landing trajectory to be generated even when straight flight is not possible. ${ }^{5}$ The sequence of alternating turning arcs requires only that the aircraft be capable of left or right turns of two different turning radii. The TDV solver analytically constructs a sequence of alternate turning arcs between initial state and the approach end of the landing runway. However, in our previous work we presumed transitions between turning arcs were instantaneous as a simplification consistent with the basic Dubins solution. We also have extended our TDV formulation in this paper to comprehensively handle cases in which initial and final waypoints are separated by arbitrarily large or small distances. In cases where performance is degraded to the extent that straight flight is not possible, it is likely the aircraft would require a nontrivial transition interval between the two constant-radius trim states sequenced in the landing trajectory. This paper mathematically describes the existence and the uniqueness of a sequence of alternating extreme turning arcs, and the transitions between alternate turning arcs for the TDV. Similar to our previous work, this sequence is developed to connect the initial state and the approach end of the landing runway, presuming constant altitude (2D) to simplify geometric analysis. Scenarios in which the aircraft cannot maintain straight flight can result from a variety of conditions such as structural damage (e.g., to a wing) or actuator failures (e.g., stuck, fully-deflected rudder or ailerons).

Other researchers have begun to design flight management architectures that will assist the pilot in decision-making during emergencies. ${ }^{6}$ Researchers have also studied the aircraft trajectory planning problem for a variety of applications, including recent work on sequencing circular segments to allow a laser to consistently track a target. ${ }^{7}$ The classic engine-out (loss-of-thrust) scenario was addressed in our previous work by an extension of a Dubins path solver ${ }^{1}$ and has also been studied in the context of a turn-back landing cast in an optimal control framework. ${ }^{8}$ There also have been efforts to design multi-layer autonomous flight management systems for Unmanned Air Systems (UAS) such as the multi-layer intelligent control architecture. $^{9}$ We have previously modeled emergency situations ranging from loss of thrust ${ }^{1}$ to actuator failures $^{2}$ to a commercial transport with severe left wing damage. ${ }^{3}$ This work adopts the same framework as was introduced in our previous work, ${ }^{1}$ as shown in Figure 1. In the presence of failures and/or damage, the emergency flight planner activates the Adaptive Flight Planner (AFP) that generates an alternative flight plan through a variable autonomy pilot interface and flight plan monitor. Within the AFP, a Landing Site Search (LSS) module determines a safe landing site, currently a runway deemed feasible based on length, width, wind conditions, etc. ${ }^{1}$ Using an identified sufficient stable trim state set, the Segmented Trajectory Planner, inspired by the maneuver automaton, ${ }^{10}$ constructs a sequence of valid post-failure trim states to this landing site. This paper presents an analytic trajectory planner that guarantees the smooth transition for the TDV, and eliminates the requirement to use a computationally-intensive iterative solver. The rest of this paper is organized as follows. Section II describes the geometric constraints required to connect the initial turning flight segment with the final turn to touchdown by considering alternate turning arcs. Section III presents criteria by which a chosen landing site is feasible(reachable) with a TDV trajectory and the existence and the uniqueness of a sequence of alternate turning arcs. Section IV characterizes the minimum number of alternate turning arcs required to progress to the feasible runway. Section V illustrates 
the extension of the geometric constraints for a sequence of alternate turning arcs with transition. Examples of TDV trajectories with transitions are examined in Section VI.

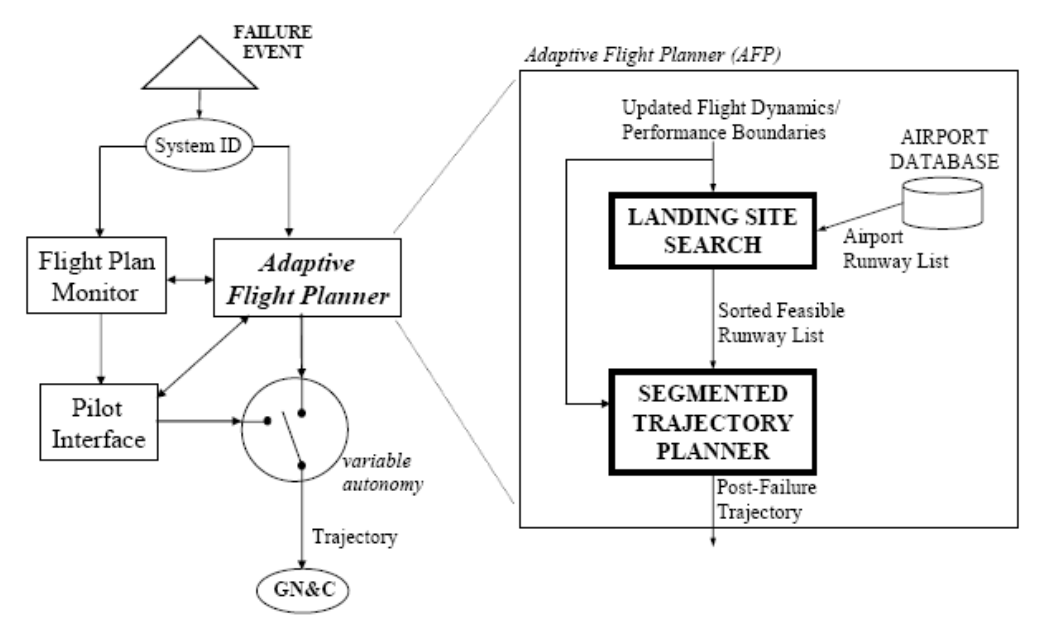

Figure 1. Emergency Flight Management Architecture.

\section{Geometric Analysis of a Sequence of Alternate Turning Arcs}

We consider the Cartesian coordinate system $X Y Z$ fixed in an inertial frame where $\hat{I}, \hat{J}$, and $\hat{K}$ are unit vectors fixed in the $X Y Z$ system. In previous work, ${ }^{5}$ we defined the concept of a Turning Dubins Vehicle as an extension of the Dubins path landing solution as follows:

Definition (Turning Dubins Vehicle (TDV)) A Turning Dubins Vehicle is a planar vehicle that is constrained to move along paths of curvature bounded both above and below, without reversing direction and maintaining a constant speed.

Let $\sigma:[0, T] \rightarrow \mathbb{R}^{2}$ be a curve for the TDV that is twice differentiable for maneuver times $T \geq 0$, and let $\mathcal{C}(s)$ be a natural representation of $\sigma$ with respect to $c_{1}$ where $c_{1}$ represents a center of the initial circular trajectory arc followed by the TDV. For TDV velocity $\vec{V}$ and unit tangent $\vec{T}=\frac{\vec{V}}{\|\vec{V}\|}$, the curvature vector $\vec{k}$ with respect to $c_{1}$ is defined as the rate of change of $\vec{T}$ with respect to arc length $s$ :

$$
\begin{aligned}
\vec{k} & =\frac{d \vec{T}}{d s}=\frac{1}{\|\vec{V}\|} \dot{\vec{T}} \\
k & =\|\vec{k}\|=\frac{1}{r}
\end{aligned}
$$

where $r$ is the turning circle radius. Since $r_{m} \leq r \leq r_{M}$ where $r_{m}$ is the minimum turning radius and $r_{M}$ is the maximum turning radius, the magnitude of the curvature of $\sigma$ is bounded above by $\frac{1}{r_{m}}$ and bounded below by $\frac{1}{r_{M}}$. Note that $\vec{k}$ is orthogonal to $\vec{T}$. Let $\Sigma$ represent the set of possible curves for the TDV, i.e., $\Sigma=\left\{\sigma \mid k \in\left[\frac{1}{r_{M}}, \frac{1}{r_{m}}\right]\right\}$.

We use $\mathcal{O}$ to denote a circle because our landing solution requires only left or right turns of two different radii. Given a center $c$ in $\mathbb{R}^{2}$, a radius $r$, and a sign of the turning rate $\operatorname{sgn}(\dot{\psi})$, let $\mathcal{O}(c, r, \operatorname{sgn}(\dot{\psi}))$ : 
$\left[0, T_{\mathcal{O}}\right] \rightarrow \mathbb{R}^{2}$ represent a circle of radius $r$ with center $c$ and direction of motion sgn $(\dot{\psi})$ where $T_{\mathcal{O}}$ denotes the maneuver time during $\mathcal{O}$ and let $\Sigma_{c}$ be the set of circular curves for the TDV as follows:

$$
\Sigma_{c}=\left\{\mathcal{O}(c, r, \operatorname{sgn}(\dot{\psi})) \mid \quad r_{m} \leq r \leq r_{M}, \quad \operatorname{sgn}(\dot{\psi})=\left\{\begin{array}{cl}
+1 & \text { if } \dot{\psi}>0 \\
-1 & \text { if } \dot{\psi}<0
\end{array}\right\}\right.
$$

$\mathcal{O}_{1}$ and $\mathcal{O}_{f}$ in $\Sigma_{c}$ represent the initial and final circular curves, respectively, and would formerly have represented the initial and final arcs from which a connecting (straight) tangent would have been computed for a Dubins path solution. Let $\mathcal{R}_{\mathcal{O}}$ be the set of possible reference circles having $\overline{c_{1} c_{f}}$ as a chord. Then

$$
\mathcal{R}_{\mathcal{O}}=\left\{\mathcal{O}\left(O, r_{r}, \operatorname{sgn}(\dot{\psi})\right) \mid \vec{r}_{O}=\frac{1}{2}\left(\vec{r}_{c_{1}}+\vec{r}_{c_{f}}\right)+\lambda\left(\cos \zeta_{O} \hat{I}+\sin \zeta_{O} \hat{J}\right), \quad \lambda \in \mathbb{R}\right\}
$$

where $\vec{r}_{c_{1}} \times \vec{r}_{c_{f}}=x_{c_{1} \times c_{f}} \hat{I}+y_{c_{1} \times c_{f}} \hat{J}+k_{c_{1} \times c_{f}} \hat{K}$ and $\zeta_{O}=\arctan \left(-\frac{x_{c_{f} c_{1}}}{y_{c_{f} c_{1}}}\right)$ where $\vec{r}_{c_{f} c_{1}}=x_{c_{f} c_{1}} \hat{I}+y_{c_{f} c_{1}} \hat{J}$.

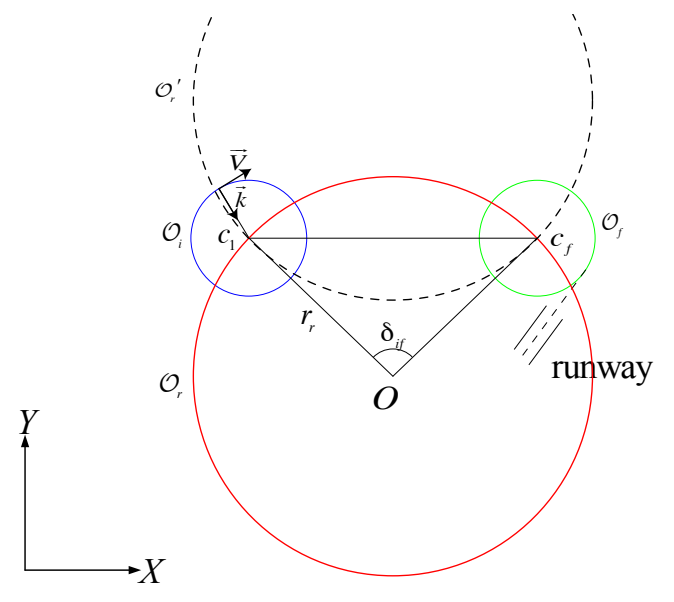

Figure 2. Reference Circle $\mathcal{O}_{r}$ having the chord $\overline{c_{1} c_{f}}$

Note that there also exists a dual reference circle $\mathcal{O}_{r}^{\prime} \in \mathcal{R}_{\mathcal{O}}$ because the direction of the unit vector representing the perpendicular bisector can be reversed, as shown in Figure 2. Additionally, a reference arc can be followed by alternating segments of two different turning radii that include a predefined safety factor sufficient for disturbance rejection. ${ }^{5}$ Let $\mathcal{O}_{r} \in \mathcal{R}_{\mathcal{O}}$, and let $r_{r}$ be a radius of a reference circle $\mathcal{O}_{r}$. Let $\delta_{i f}$ represent the radian measure of the central angle corresponding to the chord of length $\left\|\vec{r}_{c_{1} c_{f}}\right\|$. Then

$$
\delta_{1 f}=2 \arcsin \left(\frac{\left\|\vec{r}_{c_{1} c_{f}}\right\|}{2 r_{r}}\right)
$$

For a given center $c$ in $\mathbb{R}^{2}$ and two given points $p_{i}$ and $p_{f}$ in $\mathbb{R}^{2}$, let $a\left(c, p_{i}, p_{f}\right):\left[0, T_{a}\right] \rightarrow \mathbb{R}^{2}$ be a circular arc connecting $p_{i}$ and $p_{f}$ with arc center $c$.Let $c_{0}$ be a point located on a chord of length $r_{1}-r_{2}$ from $c_{1}$ in $\mathcal{O}_{r}$, and let $p_{0}$ be an intersection between $\mathcal{O}_{1}$ and a ray of the chord $\overline{c_{1} c_{0}}$ from $c_{1}$. The following theorem presents how to specify a sequence of two different turning radii for the TDV.

Theorem II.1 Let $\mathcal{O}_{r}$ be in $\mathcal{R}_{\mathcal{O}}$, and let $\mathcal{O}_{1}\left(c_{1}, r_{1}, \operatorname{sgn}(\dot{\psi})\right), \mathcal{O}_{2}\left(c_{2}, r_{2}, \operatorname{sgn}(\dot{\psi})\right)$ and $\mathcal{O}_{3}\left(c_{3}, r_{1}, \operatorname{sgn}(\dot{\psi})\right)$ be in $\Sigma_{c}$ with $r_{1}>r_{2}$. If $c_{i+1}$ is located on a chord of length $r_{1}-r_{2}$ from $c_{i}$ in $\mathcal{O}_{r}$ and distinct from $c_{i-1}$ for all $i \in\{1,2\}$, then $\mathcal{O}_{1}$ and $\mathcal{O}_{2}$ are tangent at an intersection between $\mathcal{O}_{1}$ and a ray of the chord $\overline{c_{1} c_{2}}$ from $c_{1}$, and $\mathcal{O}_{2}$ and $\mathcal{O}_{3}$ are tangent at an intersection between $\mathcal{O}_{3}$ and a ray of the chord $\overline{c_{3} c_{2}}$ from $c_{3}$. Moreover, a central angle $\delta_{12}$ subtended by the chord $\overline{c_{1} c_{2}}$ is given by:

$$
\delta_{12}=2 \arcsin \frac{r_{1}-r_{2}}{2 r_{r}}=2 \arcsin \frac{k_{r}\left(r_{1}-r_{2}\right)}{2}
$$

where $k_{r}:=1 / r_{r}$ is the curvature of the reference circle. 
Proof Let $i \in\{1,2\}$. Assume $c_{i+1}$ is located on a chord of length $r_{1}-r_{2}$ from $c_{i}$ in $\mathcal{O}_{r}$ and distinct from $c_{i-1}$. Let $p_{1}$ and $p_{2}$ be intersections between $\mathcal{O}_{1}$ and a ray of the chord $\overline{c_{1} c_{2}}$ from $c_{1}$, and between $\mathcal{O}_{3}$ and a ray of the chord $\overline{c_{3} c_{2}}$ from $c_{3}$, respectively. Since the distance between the centers of $\mathcal{O}_{1}$ and $\mathcal{O}_{2}$ is equal to the difference of the radii, $\mathcal{O}_{1}$ and $\mathcal{O}_{2}$ are tangent at $p_{1}$, and $\mathcal{O}_{2}$ and $\mathcal{O}_{3}$ are tangent at $p_{2} .{ }^{11}$ Let $\delta_{12}$ be the radian measure of the central angle corresponding to the chord $\overline{c_{1} c_{2}}$. Since the perpendicular bisector of the chord passes through the center of the reference circle $\mathcal{O}_{r}$ and the length of the chord $\overline{c_{1} c_{2}}$ is $r_{1}-r_{2}, \delta_{12}$ is given by Eq. 6 .

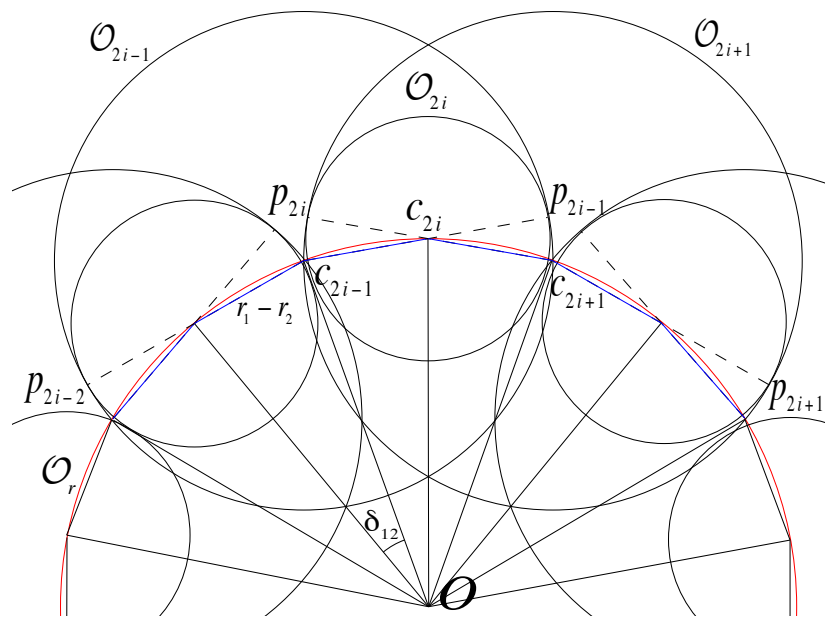

Figure 3. Product of $\mathbf{n}$ arcs

Corollary II.2 Let $\mathcal{O}_{r}$ be in $\mathcal{R}_{\mathcal{O}}$, and let $\mathcal{O}_{2 i-1}\left(c_{2 i-1}, r_{1}\right.$, sgn $\left.(\dot{\psi})\right), \mathcal{O}_{2 i}\left(c_{2 i}, r_{2}, \operatorname{sgn}(\dot{\psi})\right)$ and $\mathcal{O}_{2 i+1}$ $\left(c_{2 i+1}, r_{1}, \operatorname{sgn}(\dot{\psi})\right)$ be in $\Sigma_{c}$ with $r_{1}>r_{2}$ for each $i \in \mathbb{N}$. If $c_{i+1}$ is located on a chord of length $r_{1}-r_{2}$ from $c_{i}$ in $\mathcal{O}_{r}$ and distinct from $c_{i-1}$ for all $i \in \mathbb{N}$, then $\mathcal{O}_{2 i-1}$ and $\mathcal{O}_{2 i}$ are tangent at an intersection between $\mathcal{O}_{2 i-1}$ and a ray $\overrightarrow{c_{2 i-1} c_{2 i}}$, and $\mathcal{O}_{2 i}$ and $\mathcal{O}_{2 i+1}$ are tangent at an intersection between $\mathcal{O}_{2 i+1}$ and a ray $\overrightarrow{c_{2 i+1} c_{2 i}}$ for all $i \in \mathbb{N}$. Moreover, the central angle subtended by the chord $\overline{c_{1} c_{2 i}}$ is $(2 i-1) \delta_{12}$.

Proof Let $i \in \mathbb{N}$. Assume $c_{i+1}$ is located on a chord of length $r_{1}-r_{2}$ from $c_{i}$ in $\mathcal{O}_{r}$ and distinct from $c_{i-1}$. Let $p_{2 i-1}$ and $p_{2 i}$ represent intersections between $\mathcal{O}_{2 i-1}$ and a ray $\overrightarrow{c_{2 i-1} c_{2 i}}$, and between $\mathcal{O}_{2 i+1}$ and a ray $\overrightarrow{c_{2 i+1} c_{2 i}}$. By Theorem II.1, $\mathcal{O}_{1}$ and $\mathcal{O}_{2}$ are tangent at $p_{1}$, and $\mathcal{O}_{2}$ and $\mathcal{O}_{3}$ are tangent at $p_{2}$. Let the $n$th proposition be that $\mathcal{O}_{2 n-1}$ and $\mathcal{O}_{2 n}$ are tangent at $p_{2 n-1}$, and $\mathcal{O}_{2 n}$ and $\mathcal{O}_{2 n+1}$ are tangent at $p_{2 n}$. Suppose our $n$th proposition is true. Since chords $\overline{c_{2 n+1} c_{2 n+2}}$ and $\overline{c_{2 n+2} c_{2 n+3}}$ of the reference circle $\mathcal{O}_{r}$ have length $r_{1}-r_{2}$ by assumption, the $(n+1)$ th proposition holds. By induction, $\mathcal{O}_{2 i-1}$ and $\mathcal{O}_{2 i}$ are tangent at $p_{2 i-1}$, and $\mathcal{O}_{2 i}$ and $\mathcal{O}_{2 i+1}$ are tangent at $p_{2 i}$ for all $i \in \mathbb{N}$.

For $i=1$, it is true by Theorem II.1 that the central angle subtended by the chord $\overline{c_{1} c_{2}}$ is given by Eq. 6 . Suppose our $n$th proposition is true. Since the perpendicular bisector of the chord passes thought the center of the reference circle $\mathcal{O}_{r}$, and chords $\overline{c_{2 n+1} c_{2 n+2}}$ and $\overline{c_{2 n+2} c_{2 n+3}}$ of the reference circle $\mathcal{O}_{r}$ have length $r_{1}-r_{2}$ by assumption, Theorem II.1 holds for $\mathcal{O}_{2 n+1}\left(c_{2 n+1}, r_{1}, \operatorname{sgn}(\dot{\psi})\right), \mathcal{O}_{2 n+2}\left(c_{2 n+2}, r_{2}, \operatorname{sgn}(\dot{\psi})\right)$ and $\mathcal{O}_{2 n+3}\left(c_{2 n+3}, r_{1}, \operatorname{sgn}(\dot{\psi})\right)$. By assumption, $\overline{c_{i} c_{i+1}}$ is a chord of $\mathcal{O}_{r}$ with length $r_{1}-r_{2}$, and the central angle subtended by the chord $\overline{c_{i} c_{i+1}}$ is given by Eq. 6 for all $i \in \mathbb{N}$. Then a central angle subtended by the chord $\overline{c_{1} c_{2 n+2}}$ is $(2 n+1) \delta_{12}$. By induction, a central angle subtended by the chord $\overline{c_{1} c_{2 i}}$ is $(2 i-1) \delta_{12}$ for all $i \in \mathbb{N}$.

When we determine alternating segments of two different turning radii, the definition of a product of two arcs is used. ${ }^{12}$ Let $a_{2 i-1}$ be a circular arc of $\mathcal{O}_{2 i-1}$ such that $a_{2 i-1}\left(c_{2 i-1}, p_{2 i-2}, p_{2 i-1}\right):\left[0, T_{2 i-1}-T_{2 i-2}\right] \rightarrow$ $\mathbb{R}^{2}$, and let $a_{2 i}$ be a circular arc of $\mathcal{O}_{2 i}$ such that $a_{2 i}\left(c_{2 i}, p_{2 i-1}, p_{2 i}\right):\left[0, T_{2 i}-T_{2 i-1}\right] \rightarrow \mathbb{R}^{2}$. Since $\mathcal{O}_{2 i-1}$ and $\mathcal{O}_{2 i}$ are tangent at $p_{2 i-1}$ and $a_{2 i-1}\left(T_{2 i-1}-T_{2 i-2}\right)=a_{2 i}(0)=p_{2 i-1}$ for all $i \in \mathbb{N}$ by Corollary II.2, products 
$b_{i}$ of two arcs, $a_{2 i-1}$ and $a_{2 i}$, are defined as follows:

$$
b_{i}=a_{2 i-1} * a_{2 i}= \begin{cases}a_{2 i-1}\left(t-T_{2 i-2}\right), & T_{2 i-2} \leq t \leq T_{2 i-1} \\ a_{2 i}\left(t-T_{2 i-1}\right), & T_{2 i-1} \leq t \leq T_{2 i}\end{cases}
$$

where $T_{0}=0$. Since $\mathcal{O}_{2 i}$ and $\mathcal{O}_{2 i+1}$ are tangent at $p_{2 i}$ and $b_{i}\left(T_{2 i}-T_{2 i-2}\right)=b_{i+1}(0)=p_{2 i}$ where $b_{i}=$ $a_{2 i-1} * a_{2 i}$ and $b_{i+1}=a_{2 i+1} * a_{2(i+1)}$ for all $i \in \mathbb{N}$, then we define a product of two products as :

$$
b_{i} * b_{i+1}= \begin{cases}b_{i}\left(t-T_{2 i-2}\right), & T_{2 i-2} \leq t \leq T_{2 i} \\ b_{i+1}\left(t-T_{2 i}\right), & T_{2 i} \leq t \leq T_{2 i+2}\end{cases}
$$

where $T_{0}=0$. Therefore, $b_{1} * b_{2} * \cdots * b_{i}$ is defined for all $i \in \mathbb{N}$.

\section{Existence and Uniqueness of a Sequence of Alternate Turning Arcs}

In this section, we present criteria by which we can guarantee a TDV path from an initial state to the chosen landing site exists and is unique. The TDV trajectory is defined with respect to a particular reference circle $\mathcal{O}_{r}$. Let $a_{r}$ be an arc of the chord $\overline{c_{1} c_{f}}$ passing through $c_{i}$ where $i \in\{2,3, \cdots, 2 n\}$. Let $\left\{b_{i} \mid b_{i}=a_{2 i-1} * a_{2 i}, \quad i \in \mathbb{N}\right\}$ represent the set of products of alternate turning arcs for the TDV in $\mathcal{O}_{r} \in \mathcal{R}_{\mathcal{O}}$ such that Corollary II.2 holds, denoted by $\mathcal{B}$. In the previous sections, $\mathcal{B}$ follows an arc $a_{r}$ of $\mathcal{O}_{r}$. After $n$ sequences, however, the final circular curve $\mathcal{O}_{f}$ and the $2 n$th circular curve $\mathcal{O}_{2 n}$ for the TDV are not guaranteed tangent at $p_{2 n}$. The following theorem describes the feasibility condition about $\mathcal{O}_{r}$ for the TDV to reach the selected runway.

Theorem III.1 Let $\mathcal{O}_{r}$ be in $\mathcal{R}_{\mathcal{O}}$, and let $n \in \mathbb{N}$ be given. Suppose $\mathcal{B}=\left\{b_{i} \mid b_{i}=a_{2 i-1} * a_{2 i}, i \in\{1,2, \cdots, n\}\right\}$ in $\mathcal{O}_{r}$ such that Corollary II.2 holds. Let $\mathcal{O}_{1}\left(c_{1}, r_{1}, \operatorname{sgn}(\dot{\psi})\right)$ and $\mathcal{O}_{f}\left(c_{f}, r_{1}, \operatorname{sgn}(\dot{\psi})\right)$ represent the initial and final circular curves, respectively. Then there exists a cyclic polygon with $2 n$ edge of length $r_{1}-r_{2}$ and a edge of length $\left\|\vec{r}_{c_{f} c_{1}}\right\|$ if and only if $r_{r}$ satisfies the horizontal feasibility condition:

$$
\begin{aligned}
& \delta_{1 f}=2 n \delta_{12} \quad \text { if } 2 n \delta_{12} \leq \pi \\
& 2 \pi=2 n \delta_{12}+\delta_{1 f} \quad \text { if } \quad 2 n \delta_{12}>\pi
\end{aligned}
$$

where $\delta_{12}=2 \arcsin \left(\left(r_{1}-r_{2}\right) / 2 r_{r}\right)$ and $\delta_{1 f}=2 \arcsin \left(\left\|r_{c_{f} c_{1}}\right\| / 2 r_{r}\right)$. Therefore, the TDV can reach the selected runway.

Proof Assume there exists a cyclic polygon with $2 n$ edge of length $r_{1}-r_{2}$ and a edge of length $\left\|\vec{r}_{c_{f} c_{1}}\right\|$. Then, for each $i \in(1,2, \cdots, 2 n-1)$, line segments $\overline{c_{i} c_{i+1}}$ and $\overline{c_{2 n} c_{f}}$ are chords of length $r_{1}-r_{2}$. By Corollary II.2, the central angle subtended by the chord $\overline{c_{i} c_{i+1}}$ is given by Eq. 6 for all $i \in\{1,2, \cdots, n\}$. Since the chord $\overline{c_{2 n} c_{f}}$ has length $r_{1}-r_{2}$, a central angle of $a_{r}$ is equal to $2 n \delta_{12}$. By the definition of the reference circle, a line segment $\overline{c_{1} c_{f}}$ is a chord of length $\left\|\vec{r}_{c_{f} c_{1}}\right\|$, and the chord $\overline{c_{1} c_{f}}$ has a central angle $2 \delta_{i f}$. Suppose $2 n \delta_{12} \leq \pi$. Then the arc $a_{r}$ is a minor arc or a semicircle. Since the arc $a_{r}$ has the chord $\overline{c_{1} c_{f}}$, $r_{r}$ satisfies $2 \delta_{i f}=4 n \delta_{12}$. Suppose $2 n \delta_{12}>\pi$. Then the arc $a_{r}$ is a major arc of the chord $\overline{c_{1} c_{f}}$. Since the major and minor arc together make up the entire circle, $r_{r}$ satisfies $2 \pi=2 n \delta_{12}+\delta_{i f}$.

Assume $r_{r}$ satisfies the horizontal feasibility condition 9. Since Corollary II.2 holds, each center $c_{i}$ is joined with the next center $c_{i+1}$ by a chord of $\mathcal{O}_{r}$ with length $r_{1}-r_{2}$ for all $i \in\{1,2, \cdots, 2 n-1\}$. By the definition of the reference circle, $c_{f}$ is joined with $c_{1}$ by a chord of length $\left\|\vec{r}_{c_{f}} c_{1}\right\|$, and the central angle subtended by the chord $c_{1} c_{f}$ is equal to $\delta_{i f}$. Suppose $2 n \delta_{12} \leq \pi$. Then an arc of $a_{r}$ is a minor arc or a semicircle. Since the $\operatorname{arc} a_{r}$ has the chord $\overline{c_{1} c_{f}}$, the $\operatorname{arc} a_{r}$ has a central angle $2 n \delta_{12}$ by assumption. By Corollary II.2, the central angle subtended by the chord $\overline{c_{i} c_{i+1}}$ is given by Eq. 6 for all $i \in\{1,2, \cdots, 2 n-1\}$, and thus the central angle of the chord $\overline{c_{2 n} c_{f}}$ is equal to $\delta_{12}$. Therefore, there exists a polygon with edge lengths $\left(r_{1}-r_{2}, \cdots, r_{1}-r_{2},\left\|\vec{r}_{c_{f} c_{1}}\right\|\right)$ because congruent central angles have congruent arcs and congruent arcs have congruent chords. ${ }^{13}$ Since all its vertices of the polygon lie on a circle, there exists a cyclic polygon with with edge lengths $\left(r_{1}-r_{2}, \cdots, r_{1}-r_{2},\left\|\vec{r}_{c_{f} c_{1}}\right\|\right)$. Suppose $2 n \delta_{12}>\pi$. Then $a_{r}$ is a major arc of the chord $\overline{c_{1} c_{f}}$, and $a_{r}$ has a central angle $2 n \delta_{12}$ by assumption. By Corollary II.2, the central angle of the chord $\overline{c_{2 n} c_{f}}$ is equal to $\delta_{12}$. Therefore, there exists a cyclic polygon with $2 n$ edges of length $r_{1}-r_{2}$ and an edge of length $\left\|\vec{r}_{c_{f} c_{1}}\right\|$ because congruent central angles have congruent arcs and congruent arcs have congruent chords. ${ }^{13}$ 
Corollary III.2 A cyclic polygon as defined above is convex.

Proof Suppose $2 n \delta_{12} \leq \pi$. Then the horizontal feasibility condition is given by

$$
0=\frac{1}{2 \pi}\left(2 n \delta_{12}-\delta_{i f}\right)
$$

The left-hand side of the horizontal feasibility condition represents the winding number of the polygon. ${ }^{14} \mathrm{~A}$ sequence of signs of the central angles in the right-hand side of the above equation is given by $(1, \cdots, 1,-1)$. By the characterization theorem for the convex cyclic polygon, ${ }^{14}$ the cyclic polygon we defined above is also convex. Suppose $2 n \delta_{12}>\pi$. Then the horizontal feasibility condition is given by

$$
1=\frac{1}{2 \pi}\left(2 n \delta_{12}+\delta_{i f}\right)
$$

In this case, the winding number of the polygon is equal to 1 , and a sequence of signs of the central angles is given by $(1, \cdots, 1)$. By the same theorem, ${ }^{14}$ this cyclic polygon is convex.

Corollary III.3 A convex cyclic polygon as defined above is unique.

Proof In Theorem III.1, a convex cyclic polygon has $2 n$ edges of length $r_{1}-r_{2}$ and an edge of length $\left\|\vec{r}_{c_{f} c_{1}}\right\|$. To prove the uniqueness, we need to distinguish two cases, and it is enough to show that $r_{1}-r_{2}$ is less than $(2 n-1)\left(r_{1}-r_{2}\right)+\left\|\vec{r}_{c_{f} c_{1}}\right\|$ and $\left\|\vec{r}_{c_{f} c_{1}}\right\|$ is less than $2 n\left(r_{1}-r_{2}\right)$. Without loss of generality, let us assume that $\left\|\vec{r}_{c_{f} c_{1}}\right\|>0$. Since $n \in \mathbb{N}, n \geq 1$, and thus $(2 n-1)\left(r_{1}-r_{2}\right)+\left\|\vec{r}_{c_{f} c_{1}}\right\|>0$. Therefore, $(2 n-1)\left(r_{1}-r_{2}\right)+\left\|\vec{r}_{c_{f} c_{1}}\right\|$ and $\left\|\vec{r}_{c_{f} c_{1}}\right\|$.

Suppose $2 n \delta_{12} \leq \pi$. Then $\sin \left(\delta_{i f} / 2\right)=\sin \left(n \delta_{12}\right)$ from the horizontal feasibility condition 9. Since $0<\delta_{12} / 2<\pi / 2, \sin \left(n \delta_{12}\right)<2 n \sin \left(\delta_{12} / 2\right)$. Therefore, $\sin \left(\delta_{i f} / 2\right)<2 n \sin \left(\delta_{12} / 2\right)$. By Theorem II.1 and the definition of the reference circle, $\left\|\vec{r}_{c_{f} c_{1}}\right\| /\left(2 r_{r}\right)<2 n\left[\left(r_{1}-r_{2}\right) /\left(2 r_{r}\right)\right]$. Therefore, $\left\|\vec{r}_{c_{f} c_{1}}\right\|<2 n\left(r_{1}-r_{2}\right)$. Suppose $2 n \delta_{12}>\pi$. Then $\sin \left(\pi-\delta_{i f} / 2\right)=\sin \left(n \delta_{12}\right)$. Since we obtain the same identity as the case where $2 n \delta_{12} \leq \pi,\left\|\vec{r}_{c_{f} c_{1}}\right\|<2 n\left(r_{1}-r_{2}\right)$. By the existence and uniqueness theorem for convex cyclic polygons, ${ }^{14}$ such a cyclic polygon is unique.

\section{Determination of the Minimum Number of Alternate Turning Arcs}

Let $\mathcal{O}_{r}$ be in $\mathcal{R}_{\mathcal{O}}$. We showed that if $r_{r}$ satisfies the horizontal feasibility condition 9 , then $\left\|\vec{r}_{c_{f} c_{1}}\right\|$ is less than $2 n\left(r_{1}-r_{2}\right)$ where $n$ is the number of alternate turning arcs. Since the radius of curvature for the TDV is in $\left[r_{m}, r_{M}\right]$, the difference between two radii of $\mathcal{O}_{1}$ and $\mathcal{O}_{2}$ has the minimum value. Let $n_{m}$ represent the minimum value of $n$ in $\mathcal{B}$ for $\mathcal{O}_{r}$ satisfying the horizontal feasibility condition. In the next theorem, we consider the minimum number of alternate turning $\operatorname{arcs}$ in $\mathcal{O}_{r}$ satisfying the horizontal feasibility condition.

Theorem IV.1 Let $\mathcal{O}_{r}$ be in $\mathcal{R}_{\mathcal{O}}$ and let $n \in \mathbb{N}$ be given. Suppose $\mathcal{B}=\left\{b_{i} \mid b_{i}=a_{2 i-1} * a_{2 i}, i \in\{1,2, \cdots, n\}\right\}$ in $\mathcal{O}_{r}$ such that Corollary II.2 holds. Let $\mathcal{O}_{1}\left(c_{1}, r_{1}\right.$, sgn $\left.(\dot{\psi})\right)$ and $\mathcal{O}_{f}\left(c_{f}, r_{1}, \operatorname{sgn}(\dot{\psi})\right)$ represent the initial and final circular curves, respectively. Suppose $r_{r}$ satisfies the horizontal feasibility condition 9. Then $n_{m}=\left\lceil\frac{\left\|\vec{r}_{c_{f}} c_{1}\right\|}{2 r_{1}-2 r_{2}}\right\rceil$ if $\frac{\left\|\vec{r}_{c_{f} c_{1}}\right\|}{2 r_{1}-2 r_{2}} \notin \mathbb{N}$, and $n_{m}=\left\lceil\frac{\left\|\vec{r}_{c_{f}} c_{1}\right\|}{2 r_{1}-2 r_{2}}\right\rceil+1$ if $\frac{\left\|\vec{r}_{c_{f}} c_{1}\right\|}{2 r_{1}-2 r_{2}} \in \mathbb{N}$. Therefore, there exists a cyclic polygon with $2 n_{m}$ edges of length $r_{1}-r_{2}$ and an edge of length $\left\|\vec{r}_{c_{f} c_{1}}\right\|$ where $r_{1}=r_{M}$ and $r_{2}=r_{m}$.

Proof Suppose $r_{r}$ satisfies the horizontal feasibility condition 9. By Corollary III.3, $\left\|\vec{r}_{c_{f} c_{1}}\right\|<2 n\left(r_{1}-r_{2}\right)$. Since $r_{1}>r_{2},\left\|\vec{r}_{c_{f} c_{1}}\right\| /\left[2\left(r_{1}-r_{2}\right)\right]<n$. Let $S=\left\{n \in \mathbb{N} \mid n>\left\|\vec{r}_{c_{f} c_{1}}\right\| /\left[2\left(r_{1}-r_{2}\right)\right]\right\}$. Since $n \geq 1$ for all $n \in \mathbb{N}$, the set $S$ is bounded from below. Since $\left\|\vec{r}_{c_{f} c_{1}}\right\|<2 n\left(r_{1}-r_{2}\right)>0$, there exists $n \in \mathbb{N}$ such that $1 / n<\left\|\vec{r}_{c_{f} c_{1}}\right\|<2 n\left(r_{1}-r_{2}\right)<n .{ }^{15}$ Therefore $S$ is nonempty. Since $S \subset \mathbb{N} \subset \mathbb{R}$ and $\mathbb{R}$ is complete, the set $S$ has $\inf S \in \mathbb{R}$. Let $m=\inf S$. Suppose $m \notin S$. Then $m<s$ for all $s \in S$. We have that for $\epsilon_{1}=1$ there exists an $s^{\prime} \in S$ such that $m<s^{\prime}<m+1$. Also, we have that for $\epsilon_{2}=s^{\prime}-m>0$ there exists an $s^{\prime \prime} \in S$ such that $m<s^{\prime \prime}<s^{\prime}$. Since $s^{\prime}<m+1$, this contradicts the Peano Axioms. ${ }^{15}$ Therefore, inf $S \in S$, so $\inf S=\min S$. If $\left\|\vec{r}_{c_{f} c_{1}}\right\| /\left[2\left(r_{1}-r_{2}\right)\right]$ is not an integer, then $\min S=\left\lceil\frac{\left\|\vec{r}_{c_{f} c_{1}}\right\|}{2 r_{1}-2 r_{2}}\right\rceil$ by the definition of the ceiling function. ${ }^{16}$ Suppose $n_{s}=\left\|\vec{r}_{c_{f} c_{1}}\right\| /\left[2\left(r_{1}-r_{2}\right)\right]$ is an integer. Then $\min S=n_{s}+1$ where 
$n_{s}=\frac{\left\|\vec{r}_{c_{f} c_{1}}\right\|}{2 r_{1}-2 r_{2}}=\left\lceil\frac{\left\|\vec{r}_{c_{f} c_{1}}\right\|}{2 r_{1}-2 r_{2}}\right\rceil$ because $S=\left\{n_{s}+1, n_{s}+2, n_{s}+3, \cdots\right\}$. Since $\left\|\vec{r}_{c_{f} c_{1}}\right\|$ is given, we compute the maximum value of $r_{1}-r_{2}$. Without loss of generality, let us assume that $r_{1}=r_{M}$. Since $r_{m} \leq r_{2}<r_{M}$, $r_{1}-r_{2} \leq r_{M}-r_{m}, n_{m}=\left\lceil\frac{\left\|\vec{r}_{c_{f} c_{1}}\right\|}{2 r_{1}-2 r_{2}}\right\rceil$ if $\frac{\left\|\vec{r}_{c_{f} c_{1}}\right\|}{2 r_{1}-2 r_{2}} \notin \mathbb{N}$, and $n_{m}=\left\lceil\frac{\left\|\vec{r}_{c_{f} c_{1}}\right\|}{2 r_{1}-2 r_{2}}\right\rceil+1$ if $\frac{\left\|\vec{r}_{c_{f} c_{1}}\right\|}{2 r_{1}-2 r_{2}} \in \mathbb{N}$ where $r_{1}=r_{M}$ and $r_{2}=r_{m}$. By assumption, there exists a cyclic polygon with $2 n_{m}$ edges of length $r_{1}-r_{2}$ and an edge of length $\left\|\vec{r}_{c_{f} c_{1}}\right\|$ where $r_{1}=r_{M}$ and $r_{2}=r_{m}$.

Corollary IV.2 If circular curves of two distinct radii satisfy the condition:

$$
r_{1}=r_{M} \quad \text { and } \quad r_{2}=r_{m}
$$

then $l_{12}$ has the maximum value for all $\mathcal{O}_{r}$.

Proof Let $\mathcal{O}_{r} \in \mathcal{R}_{\mathcal{O}}$. Then $l_{12}=r_{r} 2 \delta_{12}$ where $\delta_{12}=2 \arcsin \left(\left(r_{1}-r_{2}\right) / 2 r_{r}\right)$. It is sufficient to determine the maximum value of $\delta_{12}$ for all $r_{r}$. Without loss of generality, let $r_{1}=r_{M}$. Then $\delta_{12}=\arcsin \left(\left(r_{M}-r_{2}\right) / 2 r_{r}\right)$. Since the arc sine in $\delta_{12}$ is increasing for the sine angle ranging 0 to $\pi / 2$ and $r_{1}-r_{2} \leq r_{M}-r_{m}, \delta_{12}$ has the maximum value for all $r_{r}$ if $r_{2}=r_{m}$. Therefore, $l_{12}$ has maximum value for all $r_{r}$ if $r_{1}=r_{M}$ and $r_{2}=r_{m}$. Note that since we consider all $r_{r}, r_{r}$ depends on the two centers of the initial and final circular curves.

\section{Geometric Analysis of the TDV Trajectory with Transitions}

We consider two Cartesian coordinate systems: $X Y Z$ fixed in an inertial frame and $x y z$ with origin $c_{1}$ located at the center of the first circular curve denoted by $\mathcal{O}_{1}$. The $x$ axis is taken to lie along a straight line $\overline{c_{1} p_{0}}$ in the direction of the point $p_{0}$ that represents the intersection between $\mathcal{O}_{1}$ and a ray of the chord $\overline{c_{1} c_{0}}$ from $c_{1}$, and the $y$ axis is perpendicular to the $x$ axis in the lateral plane with direction determined by the right-hand rule. $\hat{i}$ and $\hat{j}$ are unit vectors in inertial $x$ and $y$ directions, respectively, as shown in Figure 4 . For TDV velocity $\vec{V}$ and unit tangent $\vec{T}=\frac{\vec{V}}{\|\vec{V}\|}$, let $\psi$ represent the angle from unit vector $\hat{i}$ to unit tangent $\vec{T}$. Then the unit tangent $\vec{T}$ and the principal normal unit vector $\vec{n}$ with respect to $c_{1}$ are given by

$$
\begin{aligned}
& \vec{T}=\cos \psi \hat{i}+\sin \psi \hat{j} \\
& \vec{n}=\left\{\begin{array}{ccc}
-\sin \psi \hat{i}+\cos \psi \hat{j} & \text { if } & \operatorname{sgn}(\dot{\psi}) \geq 0 \\
\sin \psi \hat{i}-\cos \psi \hat{j} & \text { if } & \operatorname{sgn}(\dot{\psi})<0
\end{array}\right.
\end{aligned}
$$

In the previous sections, we defined the set of products of alternate turning arcs in $\mathcal{O}_{r}$, denoted by $\mathcal{B}$, to determine a sequence connecting an initial displacement of the TDV with the approach end of the landing runway. The set $\mathcal{B}$ includes elements $b_{i}$ which is defined by connection of two different turning flight segments. To consider a nontrivial transition between constant-radius trim states, we extend a product of two different turning arcs to a product of a turning arc and a related transition arc in the next theorem. To smoothly change radius of curvature of a sequence of alternate turning arcs, we next define transitions for the TDV.

Definition (TDV Transition) A transition for the TDV is a smooth change in radius of curvature from $r_{1}$ to $r_{2}$, or from $r_{2}$ to $r_{1}$ where $r_{1}$ and $r_{2}$ are in $\left[r_{m}, r_{M}\right]$ with $r_{1} \neq r_{2}$.

Definition (TDV Transition Arc) Let $p_{i}$ and $p_{f}$ be in $\mathbb{R}^{2}$, and $r_{1}$ and $r_{2}$ be in $\left[r_{m}, r_{M}\right]$ with $r_{1} \neq r_{2}$. Then a transition arc for the TDV is a smooth curve connecting $p_{i}$ and $p_{f}$ with a rate of change of the radius of the curvature from $r_{1}$ to $r_{2}$ or from $r_{2}$ to $r_{1}$, denoted by $\mathrm{T}_{12}$ or $\mathrm{T}_{21}$, respectively, as follows:

$$
\begin{array}{ll}
\mathrm{T}_{12}\left(M_{\mathrm{T}_{12}}, p_{i}, p_{f}\right) & :\left[0, T_{\mathrm{T}_{12}}\right] \rightarrow \mathbb{R}^{2} \\
\mathrm{~T}_{21}\left(M_{\mathrm{T}_{21}}, p_{i}, p_{f}\right) & : \quad\left[0, T_{\mathrm{T}_{21}}\right] \rightarrow \mathbb{R}^{2}
\end{array}
$$

where $M_{\mathrm{T}_{12}}$ and $M_{\mathrm{T}_{21}}$ are rates of change of the radius of the curvature for the TDV with respect to $s$ over $\mathrm{T}_{12}$ and $\mathrm{T}_{21}$, respectively.

Let $s_{0}$ be the initial natural parameter of $\sigma$. At $s_{0}$, the TDV starts its first circular turning segment. Let $s_{1}$, $s_{\mathrm{T}_{12}}, s_{2}$, and $s_{\mathrm{T}_{21}}$ represent natural parameters of $\sigma$ over the first TDV turning sequence including transition 
arcs. Figure 4 illustrates TDV turning sequences, including an example time history of curvature radius illustrating our piecewise linear transition model. Note that the instantaneous transition case would be depicted with infinite transition slope, or transition slope may be sufficiently low to preclude reaching the designated minimum turn radius. The following theorem guarantees the existence and uniqueness for a space curve of $a_{1} * \mathrm{~T}_{12} * a_{2} * \mathrm{~T}_{21}$ where two transitions connect the first and the second circular turning segments $a_{1}$ and $a_{2}$, respectively.

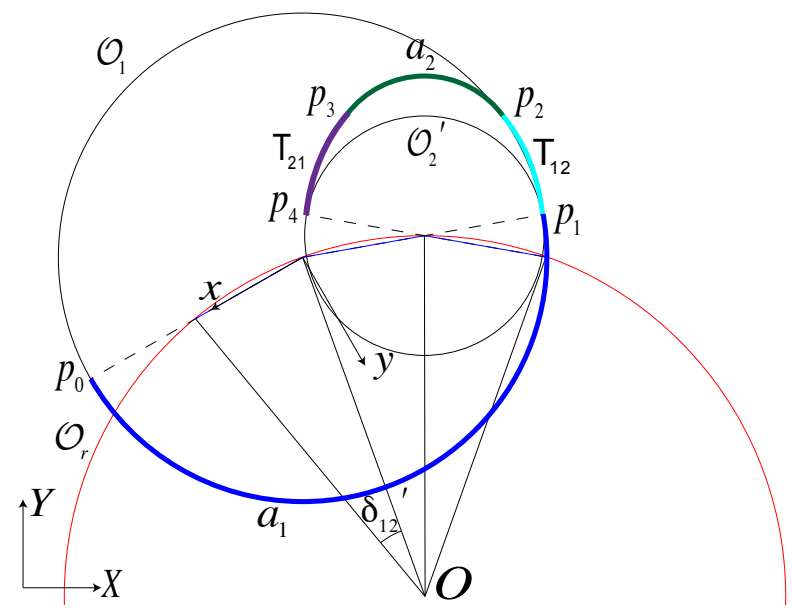

(a) Alternate Turning Arcs with Transition when sgn $(\dot{\psi})>0$

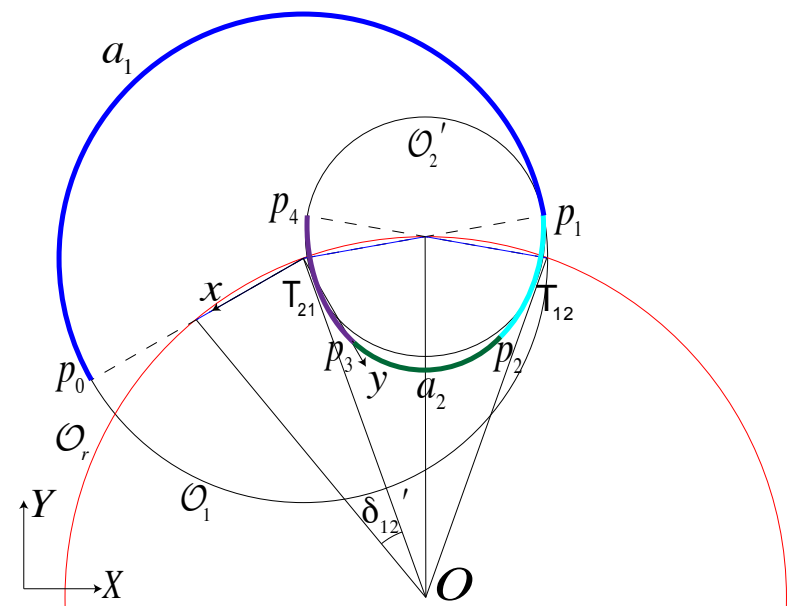

(b) Alternate Turning Arcs with Transition when sgn $(\dot{\psi})<0$

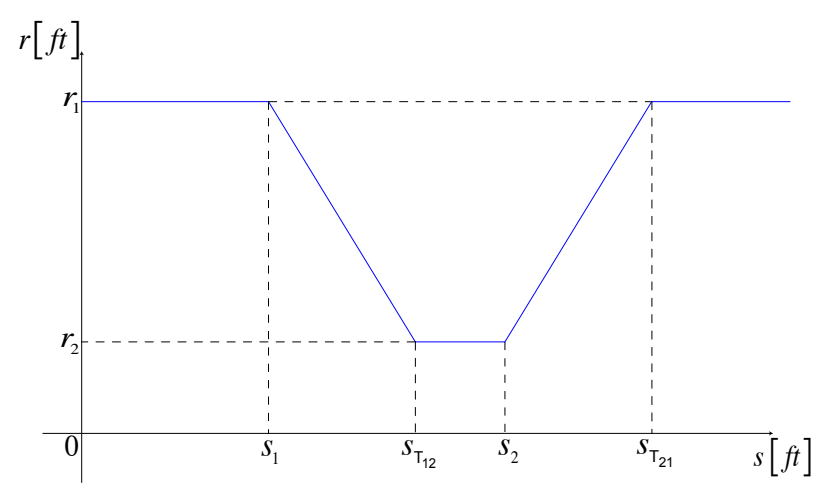

(c) Radius of Curvature for the TDV on $s_{0} \leq s \leq s_{\mathrm{T}_{21}}$

Figure 4. Alternate Turning Arcs with Transition

Theorem V.1 Let $\mathcal{O}_{r}$ be in $\mathcal{R}_{\mathcal{O}}$, and let $\mathcal{O}_{1}\left(c_{1}, r_{1}\right.$, sgn $\left.(\dot{\psi})\right)$ and $\mathcal{O}_{2}\left(c_{2}, r_{2}\right.$, sgn $\left.(\dot{\psi})\right)$ be in $\Sigma_{c}$ with $r_{1} \neq r_{2}$. Let $a_{1}$ be a circular arc of $\mathcal{O}_{1}$ with center $c_{1}$ such that $a_{1}\left(c_{1}, p_{0}, p_{1}\right):\left[0, T_{1}\right] \rightarrow \mathbb{R}^{2}$ where $\vec{r}_{p_{0}}=\vec{r}_{c_{1}}+\mathcal{C}\left(s_{0}\right)$, and $\vec{r}_{p_{1}}=\vec{r}_{c_{1}}+\mathcal{C}\left(s_{1}\right)$. Let $a_{2}$ be a circular arc of $\mathcal{O}_{2}$ with center $c_{2}$ such that $a_{2}\left(c_{2}, p_{2}, p_{3}\right)$ : $\left[0, T_{2}-T_{\mathrm{T}_{12}}\right] \rightarrow \mathbb{R}^{2}$ where $\vec{r}_{p_{2}}=\vec{r}_{c_{1}}+\mathcal{C}\left(s_{\mathrm{T}_{12}}\right)$ and $\vec{r}_{p_{3}}=\vec{r}_{c_{1}}+\mathcal{C}\left(s_{2}\right)$. Suppose $M_{\mathrm{T}_{12}}$ is constant and $M_{\mathrm{T}_{21}}=-M_{\mathrm{T}_{12}}$. If the radius of curvature for the $T D V, r(s)$, is given by:

$$
r(s)=\left\{\begin{array}{ccc}
r_{1} & \text { if } & s_{0} \leq s \leq s_{1} \\
M_{\mathrm{T}_{12}}\left(s-s_{1}\right)+r_{1} & \text { if } & s_{1} \leq s \leq s_{\mathrm{T}_{12}} \\
r_{2} & \text { if } & s_{\mathrm{T}_{12}} \leq s \leq s_{2} \\
M_{\mathrm{T}_{21}}\left(s-s_{2}\right)+r_{2} & \text { if } & s_{2} \leq s \leq s \mathrm{~T}_{21}
\end{array}\right.
$$

then the curvature $k(s)$ is continuous on $s_{0} \leq s \leq s \mathrm{~T}_{21}$. Therefore, there exists one and only one $a_{1} * \mathrm{~T}_{12} *$ $a_{2} * \mathrm{~T}_{21}$ having the curvature $k(s)$ along $\sigma$ where $\mathrm{T}_{12}\left(M_{\mathrm{T}_{12}}, p_{1}, p_{2}\right):\left[0, T_{\mathrm{T}_{12}}-T_{1}\right] \rightarrow \mathbb{R}^{2}$ on $\left[s_{1}, s \mathrm{~T}_{\mathrm{T}_{12}}\right]$ and 
$\mathrm{T}_{21}\left(M_{\mathrm{T}_{21}}, p_{3}, p_{4}\right):\left[0, T_{\mathrm{T}_{21}}-T_{2}\right] \rightarrow \mathbb{R}^{2}$ on $\left[s_{2}, s_{\mathrm{T}_{21}}\right]$.

Proof Assume the radius of curvature for the TDV, $r(s)$, satisfies Eq. 17 on $\left[s_{0}, s_{T_{21}}\right]$ as shown in Figure 4(c). $r(s)$ is the reciprocal of the curvature, thus the curvature of $\sigma$ is given by:

$$
k(s)=\frac{1}{r(s)}=\left\{\begin{array}{ccc}
\frac{1}{r_{1}} & \text { if } & s_{0} \leq s \leq s_{1} \\
\frac{1}{M_{\mathrm{T}_{12}}\left(s-s_{1}\right)+r_{1}} & \text { if } & s_{1} \leq s \leq s \mathrm{~T}_{12} \\
\frac{1}{r_{2}} & \text { if } & s_{\mathrm{T}_{12}} \leq s \leq s_{2} \\
\frac{1}{M_{\mathrm{T}_{21}}\left(s-s_{2}\right)+r_{2}} & \text { if } & s_{2} \leq s \leq s \mathrm{~T}_{21}
\end{array}\right.
$$

¿From the Serret-Frenet equations of a plane curve, ${ }^{17} \vec{T}$ and $\vec{n}$ along curve $\mathcal{C}(s)$ with respect to $c_{1}$ satisfy

$$
\begin{aligned}
& \frac{d \vec{T}}{d s}=k \vec{n} \\
& \frac{d \vec{n}}{d s}=-k \vec{T}
\end{aligned}
$$

If $\operatorname{sgn}(\dot{\psi})>0$, then $\frac{d \vec{T}}{d s}=\frac{d \psi}{d s}(-\sin \psi \hat{i}+\cos \psi \hat{j})=\frac{d \psi}{d s} \vec{n}$ and $\frac{d \vec{n}}{d s}=-\frac{d \psi}{d s}(\cos \psi \hat{i}+\sin \psi \hat{j})=-\frac{d \psi}{d s} \vec{T}$ from Eqs. 13 and 14. If $\operatorname{sgn}(\dot{\psi})<0$, then $\frac{d \vec{T}}{d s}=\frac{d \psi}{d s}(-\sin \psi \hat{i}+\cos \psi \hat{j})=-\frac{d \psi}{d s} \vec{n}$ and $\frac{d \vec{n}}{d s}=\frac{d \psi}{d s}(\cos \psi \hat{i}+\sin \psi \hat{j})=$ $\frac{d \psi}{d s} \vec{T}$ from Eqs. 13 and 14. The heading angle of $\sigma$ then satisfies

$$
\psi(s)=\left\{\begin{array}{ccc}
\int k d s+\psi_{\mathcal{C}} & \text { if } & \operatorname{sgn}(\dot{\psi})>0 \\
-\int k d s+\psi_{\mathcal{C}} & \text { if } & \operatorname{sgn}(\dot{\psi})<0
\end{array}\right.
$$

where $\psi_{C}$ represents a constant of integration in Eq. 21 along $\sigma$. Let $\psi_{0}$ be an initial heading angle of the TDV with respect to $c_{1}$. Then $\psi_{0}=\frac{\pi}{2}$ if $\operatorname{sgn}(\dot{\psi})>0$, and $\psi_{0}=\frac{3 \pi}{2}$ if $\operatorname{sgn}(\dot{\psi})<0$. Let $\psi_{1}, \psi_{\mathrm{T}_{12}}, \psi_{2}$, and $\psi_{\mathrm{T}_{21}}$ represent the constants of integration in Eq. 21 along $\sigma$ over segments $\left[s_{0}, s_{1}\right],\left[s_{1}, s_{\mathrm{T}_{12}}\right],\left[s_{\mathrm{T}_{12}}, s_{2}\right]$, and $\left[s_{2}, s_{\mathrm{T}_{21}}\right]$, respectively, with respect to $c_{1}$. Substituting Eq. 18 into Eq. 21 and integrating with respect to $s$ : If $\operatorname{sgn}(\dot{\psi})>0$, then

$$
\begin{aligned}
& \psi(s)=\int_{s_{0}}^{s_{\mathrm{T}_{21}}} k d \tau+\psi_{C} \\
& =\left\{\begin{array}{lll}
\int_{s_{0}}^{s} \frac{1}{r_{1}} d \tau+\psi_{0}=\frac{1}{r_{1}}\left(s-s_{0}\right)+\psi_{0} & \text { if } \quad s_{0} \leq s \leq s_{1} \\
\int_{s_{1}}^{s} \frac{1}{M_{\mathrm{T}_{12}}\left(\tau-s_{1}\right)+r_{1}} d \tau+\psi_{1}=\frac{1}{M_{\mathrm{T}_{12}}} \ln \left(\frac{M_{\mathrm{T}_{12}}\left(s-s_{1}\right)+r_{1}}{r_{1}}\right)+\psi_{1} & \text { if } & s_{1} \leq s \leq s_{\mathrm{T}_{12}} \\
\int_{s_{\mathrm{T}_{12}}}^{s} \frac{1}{r_{2}} d \tau+\psi_{\mathrm{T}_{12}}=\frac{1}{r_{2}}\left(s-s_{\mathrm{T}_{12}}\right)+\psi_{\mathrm{T}_{12}} & \text { if } & s_{\mathrm{T}_{12}} \leq s \leq s_{2} \\
\int_{s_{2}}^{s} \frac{1}{M_{\mathrm{T}_{21}}\left(\tau-s_{2}\right)+r_{2}} d \tau+\psi_{2}=\frac{1}{M_{\mathrm{T}_{21}}} \ln \left(\frac{M_{\mathrm{T}_{21}}\left(s-s_{2}\right)+r_{2}}{r_{2}}\right)+\psi_{2} & \text { if } & s_{2} \leq s \leq s_{\mathrm{T}_{21}}
\end{array}\right.
\end{aligned}
$$

If $\operatorname{sgn}(\dot{\psi})<0$,

$$
\begin{aligned}
& \psi(s)=\int_{s_{0}}^{s \mathrm{~T}_{21}}-k d \tau+\psi_{C} \\
& =\left\{\begin{array}{lll}
\int_{s_{0}}^{s}-\frac{1}{r_{1}} d \tau+\psi_{0}=-\frac{1}{r_{1}}\left(s-s_{0}\right)+\psi_{0} & \text { if } \quad s_{0} \leq s \leq s_{1} \\
\int_{s_{1}}^{s}-\frac{1}{M_{\mathrm{T}_{12}}\left(\tau-s_{1}\right)+r_{1}} d \tau+\psi_{1}=\frac{1}{M_{\mathrm{T}_{21}}} \ln \left(\frac{M_{\mathrm{T}_{12}}\left(s-s_{1}\right)+r_{1}}{r_{1}}\right)+\psi_{1} & \text { if } \quad s_{1} \leq s \leq s_{\mathrm{T}_{12}} \\
\int_{s_{\mathrm{T}_{12}}^{s}-\frac{1}{r_{2}} d \tau+\psi_{\mathrm{T}_{12}}=-\frac{1}{r_{2}}\left(s-s_{\mathrm{T}_{12}}\right)+\psi_{\mathrm{T}_{12}}} & \text { if } \quad s s_{\mathrm{T}_{12}} \leq s \leq s_{2} \\
\int_{s_{2}}^{s}-\frac{1}{M_{\mathrm{T}_{21}}\left(\tau-s_{2}\right)+r_{2}} d \tau+\psi_{2}=\frac{1}{M_{\mathrm{T}_{12}}} \ln \left(\frac{M_{\mathrm{T}_{21}}\left(s-s_{2}\right)+r_{2}}{r_{2}}\right)+\psi_{2} & \text { if } & s_{2} \leq s \leq s_{\mathrm{T}_{21}}
\end{array}\right.
\end{aligned}
$$




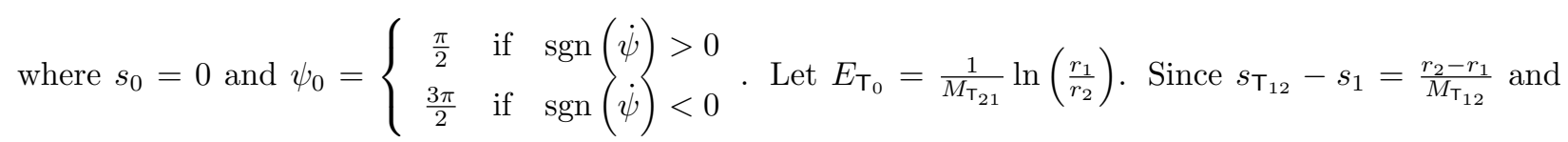
$s_{\mathrm{T}_{21}}-s_{2}=\frac{r_{1}-r_{2}}{M_{\mathrm{T}_{21}}}$ from Eq. 17, heading angles over $s$ in terms of $\psi_{1}$ and $\psi_{2}$ from Eqs. 22 and 23 are given by:

$$
\left.\left.\begin{array}{l}
\psi_{\mathrm{T}_{12}}=E_{\mathrm{T}_{0}}+\psi_{1} \\
\psi_{\mathrm{T}_{21}}=E_{\mathrm{T}_{0}}+\psi_{2}
\end{array}\right\} \text { if } \operatorname{sgn}(\dot{\psi})>0, \text { and } \quad \begin{array}{l}
\psi_{\mathrm{T}_{12}}=-E_{\mathrm{T}_{0}}+\psi_{1} \\
\psi_{\mathrm{T}_{21}}=-E_{\mathrm{T}_{0}}+\psi_{2}
\end{array}\right\} \text { if } \operatorname{sgn}(\dot{\psi})<0
$$

where $\psi_{1}=\psi\left(s_{1}\right), \psi_{\mathrm{T}_{12}}=\psi\left(s_{\mathrm{T}_{12}}\right), \psi_{2}=\psi\left(s_{2}\right)$, and $\psi_{\mathrm{T}_{21}}=\psi\left(s_{\mathrm{T}_{21}}\right)$. Since $\vec{T}=\frac{d \mathcal{C}(s)}{d s}$ and $\vec{T}=\cos \psi \hat{i}+$ $\sin \psi \hat{j}$, the natural representation $\mathcal{C}(s)$ of $\sigma$ with respect to $c_{1}$ is given by

$$
\mathcal{C}(s)=\int_{s_{0}}^{s} \vec{T} d s+\mathcal{C}_{C}
$$

where $\mathcal{C}_{C}$ is a constant vector of integration along $\sigma$ with respect to $c_{1}$. By the definition of the transition arc, let $\mathrm{T}_{12}\left(M_{\mathrm{T}_{12}}, p_{1}, p_{2}\right):\left[0, T_{\mathrm{T}_{12}}-T_{1}\right] \rightarrow \mathbb{R}^{2}$ on $\left[s_{1}, s_{\mathrm{T}_{12}}\right]$, and $\mathrm{T}_{21}\left(M_{\mathrm{T}_{21}}, p_{3}, p_{4}\right):\left[0, T_{\mathrm{T}_{21}}-T_{2}\right] \rightarrow \mathbb{R}^{2}$ on $\left[s_{2}, s_{\mathrm{T}_{21}}\right]$ where $\vec{r}_{p_{4}}=\vec{r}_{c_{1}}+\vec{r}\left(s_{\mathrm{T}_{21}}\right)$. Then the products of two arcs, ${ }^{5}$ including $a_{1} * \mathrm{~T}_{12}$ and $a_{2} * \mathrm{~T}_{21}$, are defined, and thus we define a product of two products as follows:

$$
b_{1}=a_{1} * \mathrm{~T}_{12} * a_{2} * \mathrm{~T}_{21}=\left\{\begin{array}{cc}
a_{1}(t) & 0 \leq t \leq T_{1} \\
\mathrm{~T}_{12}\left(t-T_{1}\right) & T_{1} \leq t \leq T_{\mathrm{T}_{12}} \\
a_{2}\left(t-T_{\mathrm{T}_{12}}\right) & T_{\mathrm{T}_{12}} \leq t \leq T_{2} \\
\mathrm{~T}_{21}\left(t-T_{2}\right) & T_{2} \leq t \leq T_{\mathrm{T}_{21}}
\end{array}\right.
$$

where $a_{1}, \mathbf{T}_{12}, a_{2}$, and $\mathbf{T}_{21}$ are defined by the natural representation $\mathcal{C}(s)$ of $\sigma$ on $\left[s_{0}, s_{1}\right],\left[s_{1}, s{\mathrm{~T}_{12}}_{12}\right],\left[s_{\mathrm{T}_{12}}, s_{2}\right]$, and $\left[s_{2}, s_{\mathrm{T}_{21}}\right]$, respectively.

When we prove that $r(s)$ is continuous on $\left[s_{0}, s_{\mathrm{T}_{21}}\right]$, we use the $\epsilon-\delta$ property of continuity. ${ }^{15}$ Let $\epsilon>0, s_{1_{a}} \in\left[s_{0}, s_{1}\right]$, and $\delta=\epsilon$. Then $\left|s-s_{1_{a}}\right|<\delta$ implies $\left|r(s)-r\left(s_{1_{a}}\right)\right|=\left|r_{1}-r_{1}\right|=0<\epsilon$ because $r(s)$ is a constant function on $\left[s_{0}, s_{1}\right]$. Therefore, the radius of curvature for the TDV, $r(s)$, is continuous on $\left[s_{0}, s_{1}\right]$, and also on $\left[s_{\mathrm{T}_{12}}, s_{2}\right]$. Let $s_{\mathrm{T}_{12 a}} \in\left[s_{1}, s_{\mathrm{T}_{12}}\right]$ and $\delta=-\frac{\epsilon}{M_{\mathrm{T}_{12}}}$. Then $\left|s-s_{\mathrm{T}_{12 a}}\right|<\delta$ implies $\left|M_{\mathrm{T}_{12}}\left(s-s_{1}\right)+r_{1}-\left(M_{\mathrm{T}_{12}}\left(s_{\mathrm{T}_{12 a}}-s_{1}\right)+r_{1}\right)\right|=-M_{\mathrm{T}_{12}}\left|s-s_{\mathrm{T}_{12 a}}\right|<\epsilon$. Therefore, the radius of curvature for the TDV, $r(s)$, is continuous on $\left[s_{1}, s_{\mathrm{T}_{12}}\right]$, and also on $\left[s_{2}, s_{\mathrm{T}_{21}}\right]$ because $M_{\mathrm{T}_{12}}=-M_{\mathrm{T}_{21}}$. Since $\lim _{s \rightarrow s_{1}+} r(s)=r_{1}$ by the definition of transition arc $\mathrm{T}_{12}, \lim _{s \rightarrow s_{1}-} r(s)=\lim _{s \rightarrow s_{1}+} r(s)$, and then $\lim _{s \rightarrow s_{1}} r(s)=r_{1}$. Similarly, $\lim _{s \rightarrow s \mathrm{~T}_{12}} r(s)=r_{2}$, and $\lim _{s \rightarrow s_{2}} r(s)=r_{2}$ from the definitions of $\mathrm{T}_{12}$ and $\mathrm{T}_{21}$. Therefore, the radius of curvature for the TDV, $r(s)$, is continuous on $\left[s_{0}, s_{\mathrm{T}_{21}}\right]$. If $f$ and $g$ are real-valued continuous functions on any interval in $\mathbb{R}$ such that $g\left(s_{a}\right) \neq 0$ for all $s_{a}$ in the interval, then $\frac{f}{g}$ is continuous on the interval. ${ }^{15}$ Since $r_{2} \leq r(s) \leq r_{1}$ for all $s \in\left[s_{0}, s_{\mathrm{T}_{21}}\right]$, the curvature of $\sigma$ is continuous on $\left[s_{0}, s_{\mathrm{T}_{21}}\right]$. By the fundamental existence and uniqueness theorem for space curves, ${ }^{17}$ there exists one and only one $a_{1} * \mathrm{~T}_{12} * a_{2} * \mathrm{~T}_{21}$ having the curvature $k(s)$ along $\sigma$.

From sufficient condition 17 in Theorem V.1, $s_{\mathrm{T}_{12}} \leq s_{2}$. From Eqs. 22 and 23,

$$
\left|\psi_{2}-\psi_{\mathrm{T}_{12}}\right| \geq 0
$$

Substituting Eq. 24 and using $E_{\mathrm{T}_{0}}=\frac{1}{M_{\mathrm{T}_{21}}} \ln \left(\frac{r_{1}}{r_{2}}\right)$, we have the following constraints on $M_{\mathrm{T}_{21}}$ :

$$
\frac{2 \ln \left(\frac{r_{1}}{r_{2}}\right)}{\left|\psi_{\mathrm{T}_{21}}-\psi_{1}\right|} \leq M_{\mathrm{T}_{21}}
$$

If the rate of change of the radius of curvature for the TDV with respect to $s$ does not satisfy this constraint on $M_{\mathrm{T}_{21}}$, then $\psi_{2}-\psi_{\mathrm{T}_{12}}<0$ if $\operatorname{sgn}(\dot{\psi})>0$, and $\psi_{\mathrm{T}_{12}}-\psi_{2}<0$ if $\operatorname{sgn}(\dot{\psi})<0$. As a result, $s_{2} \leq s_{\mathrm{T}_{12}}$, and thus the TDV must reverse direction or the alternate arc $2 \pi-\left|\psi_{2}-\psi_{\mathrm{T}_{12}}\right|$ about $a_{2}$ should be considered. From the definition of the TDV, however, the TDV cannot reverse direction. We assume that $M_{\mathrm{T}_{21}}$ is greater than or equal to $2 \ln \left(\frac{r_{1}}{r_{2}}\right) /\left|\psi_{\mathrm{T}_{21}}-\psi_{1}\right|$. 
Let $\mathcal{O}_{r} \in \mathcal{R}_{\mathcal{O}}$. Consider $b_{1}=a_{1} * \mathrm{~T}_{12} * a_{2} * \mathrm{~T}_{21}$ in Eq. 26, as shown in Figure 4 (a) and (b). Let $\mathcal{O}_{2}^{\prime}\left(c_{2}^{\prime}, r_{2}^{\prime}\right.$, sgn $\left.(\dot{\psi})\right)$ be in $\Sigma_{c}$ where $r_{2} \leq r_{2}^{\prime} \leq r_{1}$ and let $a_{2}^{\prime}$ be a circular arc of $\mathcal{O}_{2}^{\prime}$ such that $a_{2}^{\prime}\left(c_{2}^{\prime}, p_{1}, p_{2}^{\prime}\right)$ : $\left[0, T_{2}^{\prime}-T_{1}\right] \rightarrow \mathbb{R}^{2}$. Suppose $a_{1} * a_{2}^{\prime}$ in $\mathcal{O}_{r}$ such that Theorem II.1 holds, and $p_{4}$ in the transition arc $\mathrm{T}_{21}\left(M_{\mathrm{T}_{21}}, p_{3}, p_{4}\right)$ is located at $p_{2}^{\prime}$ such that if $2 \delta_{12}^{\prime} \leq \pi$,

$$
\begin{aligned}
& \psi_{\mathrm{T}_{21}}=\psi_{0}+2 \pi+2 \delta_{12}^{\prime} \quad \text { if } \quad \frac{\operatorname{sgn}(\dot{\psi})}{\operatorname{sgn}\left(k_{c_{1} \times c_{f}}\right)}>0 \\
& \psi_{\mathrm{T}_{21}}=\psi_{0}+2 \pi-2 \delta_{12}^{\prime} \quad \text { if } \quad \frac{\operatorname{sgn}(\dot{\psi})}{\operatorname{sgn}\left(k_{c_{1} \times c_{f}}\right)}<0
\end{aligned}
$$

If $2 \delta_{12}^{\prime}>\pi$

$$
\begin{aligned}
& \psi_{\mathrm{T}_{21}}=\psi_{0}+2 \pi-2 \delta_{12}^{\prime} \text { if } \frac{\operatorname{sgn}(\dot{\psi})}{\operatorname{sgn}\left(k_{c_{1} \times c_{f}}\right)}>0 \\
& \psi_{\mathrm{T}_{21}}=\psi_{0}+2 \pi+2 \delta_{12}^{\prime} \text { if } \quad \frac{\operatorname{sgn}(\dot{\psi})}{\operatorname{sgn}\left(k_{c_{1} \times c_{f}}\right)}<0
\end{aligned}
$$

where $\delta_{12}^{\prime}=2 \arcsin \frac{r_{1}-r_{2}^{\prime}}{2 r_{r}}$ and $\psi_{0}=\left\{\begin{array}{ccc}\frac{\pi}{2} & \text { if } & \operatorname{sgn}(\dot{\psi})>0 \\ \frac{3 \pi}{2} & \text { if } & \operatorname{sgn}(\dot{\psi})<0\end{array}\right.$. From Eq. 25, the natural representation of $\sigma$ with respect to $c_{1}$ at $s_{\mathrm{T}_{21}}$ is given by:

if $\operatorname{sgn}(\dot{\psi})>0$,

$$
\begin{aligned}
\mathcal{C}_{\mathrm{T}_{21}}= & {\left[M_{\mathrm{T}_{12}} E_{\mathrm{T}_{12}^{2}} \cos \psi_{1}-M_{\mathrm{T}_{12}} E_{\mathrm{T}_{12}^{1}} \sin \psi_{1}+M_{\mathrm{T}_{12}} E_{\mathrm{T}_{12}^{2}} \cos \psi_{\mathrm{T}_{21}}+\left(M_{\mathrm{T}_{12}} E_{\mathrm{T}_{12}^{1}}+r_{1}\right) \sin \psi_{\mathrm{T}_{21}}\right] \hat{i} } \\
& +\left[M_{\mathrm{T}_{12}} E_{\mathrm{T}_{12}^{2}} \sin \psi_{1}+M_{\mathrm{T}_{12}} E_{\mathrm{T}_{12}^{1}} \cos \psi_{1}+M_{\mathrm{T}_{12}} E_{\mathrm{T}_{12}^{2}} \sin \psi_{\mathrm{T}_{21}}-\left(M_{\mathrm{T}_{12}} E_{\mathrm{T}_{12}^{1}}+r_{1}\right) \cos \psi_{\mathrm{T}_{21}}\right] \hat{j}(33)
\end{aligned}
$$

If $\operatorname{sgn}(\dot{\psi})<0$,

$$
\begin{aligned}
\mathcal{C}_{\mathrm{T}_{21}}= & {\left[M_{\mathrm{T}_{12}} E_{\mathrm{T}_{12}^{2}} \cos \psi_{1}+M_{\mathrm{T}_{12}} E_{\mathrm{T}_{12}^{1}} \sin \psi_{1}+M_{\mathrm{T}_{12}} E_{\mathrm{T}_{12}^{2}} \cos \psi_{\mathrm{T}_{21}}-\left(M_{\mathrm{T}_{12}} E_{\mathrm{T}_{12}^{1}}+r_{1}\right) \sin \psi_{\mathrm{T}_{21}}\right] \hat{i} } \\
& +\left[M_{\mathrm{T}_{12}} E_{\mathrm{T}_{12}^{2}} \sin \psi_{1}-M_{\mathrm{T}_{12}} E_{\mathrm{T}_{12}^{1}} \cos \psi_{1}+M_{\mathrm{T}_{12}} E_{\mathrm{T}_{12}^{2}} \sin \psi_{\mathrm{T}_{21}}+\left(M_{\mathrm{T}_{12}} E_{\mathrm{T}_{12}^{1}}+r_{1}\right) \cos \psi_{\mathrm{T}_{21}}\right] \hat{j}(34)
\end{aligned}
$$

where

$$
\begin{aligned}
E_{\mathrm{T}_{12}^{1}} & =\frac{r_{2}}{1+M_{\mathrm{T}_{12}}^{2}}\left[\sin E_{\mathrm{T}_{0}}+M_{\mathrm{T}_{12}} \cos E_{\mathrm{T}_{0}}-\frac{r_{1}}{r_{2}} M_{\mathrm{T}_{12}}\right] \\
E_{\mathrm{T}_{12}^{2}} & =\frac{r_{2}}{1+M_{\mathrm{T}_{12}}^{2}}\left[\cos E_{\mathrm{T}_{0}}-M_{\mathrm{T}_{12}} \sin E_{\mathrm{T}_{0}}-\frac{r_{1}}{r_{2}}\right] \\
E_{\mathrm{T}_{0}} & =\frac{1}{M_{\mathrm{T}_{21}}} \ln \left(\frac{r_{1}}{r_{2}}\right)
\end{aligned}
$$

We then obtain the following simultaneous equations:

If $\operatorname{sgn}(\dot{\psi})>0$,

$$
\begin{array}{r}
M_{\mathrm{T}_{12}} E_{\mathrm{T}_{12}^{2}} \cos \psi_{1}-M_{\mathrm{T}_{12}} E_{\mathrm{T}_{12}^{1}} \sin \psi_{1}+M_{\mathrm{T}_{12}} E_{\mathrm{T}_{12}^{2}} \cos \psi_{\mathrm{T}_{21}}+\left(M_{\mathrm{T}_{12}} E_{\mathrm{T}_{12}^{1}}+r_{1}\right) \sin \psi_{\mathrm{T}_{21}} \\
=r_{2}^{\prime} \sin \psi_{\mathrm{T}_{21}}+\left(r_{1}-r_{2}^{\prime}\right) \sin \psi_{1} \\
M_{\mathrm{T}_{12}} E_{\mathrm{T}_{12}^{2}} \sin \psi_{1}+M_{\mathrm{T}_{12}} E_{\mathrm{T}_{12}^{1}} \cos \psi_{1}+M_{\mathrm{T}_{12}} E_{\mathrm{T}_{12}^{2}} \sin \psi_{\mathrm{T}_{21}}-\left(M_{\mathrm{T}_{12}} E_{\mathrm{T}_{12}^{1}}+r_{1}\right) \cos \psi_{\mathrm{T}_{21}} \\
=-r_{2}^{\prime} \cos \psi_{\mathrm{T}_{21}}+\left(r_{2}^{\prime}-r_{1}\right) \cos \psi_{1}
\end{array}
$$


If $\operatorname{sgn}(\dot{\psi})<0$

$$
\begin{array}{r}
M_{\mathrm{T}_{12}} E_{\mathrm{T}_{12}^{2}} \cos \psi_{1}+M_{\mathrm{T}_{12}} E_{\mathrm{T}_{12}^{1}} \sin \psi_{1}+M_{\mathrm{T}_{12}} E_{\mathrm{T}_{12}^{2}} \cos \psi_{\mathrm{T}_{21}}-\left(M_{\mathrm{T}_{12}} E_{\mathrm{T}_{12}^{1}}+r_{1}\right) \sin \psi_{\mathrm{T}_{21}} \\
=-r_{2}^{\prime} \sin \psi_{\mathrm{T}_{21}}-\left(r_{1}-r_{2}^{\prime}\right) \sin \psi_{1} \\
M_{\mathrm{T}_{12}} E_{\mathrm{T}_{12}^{2}} \sin \psi_{1}-M_{\mathrm{T}_{12}} E_{\mathrm{T}_{12}^{1}} \cos \psi_{1}+M_{\mathrm{T}_{12}} E_{\mathrm{T}_{12}^{2}} \sin \psi_{\mathrm{T}_{21}}+\left(M_{\mathrm{T}_{12}} E_{\mathrm{T}_{12}^{1}}+r_{1}\right) \cos \psi_{\mathrm{T}_{21}} \\
=r_{2}^{\prime} \cos \psi_{\mathrm{T}_{21}}+\left(r_{1}-r_{2}^{\prime}\right) \cos \psi_{1}
\end{array}
$$

Suppose $\psi_{\mathrm{T}_{21}}=5 \pi / 2-2 \delta_{12}^{\prime}$. Then $\psi_{1}=3 \pi / 2-\delta_{12}^{\prime}$. Substituting into Eqs. 38 and 41 and using trigonometric identities,

$$
\begin{aligned}
& \left(M_{\mathrm{T}_{12}} E_{\mathrm{T}_{12}^{1}}+r_{1}-r_{2}^{\prime}\right) \cos \frac{\delta_{12}^{\prime}}{2} \cos \frac{3 \delta_{12}^{\prime}}{2}=-M_{\mathrm{T}_{12}} E_{\mathrm{T}_{12}^{2}} \cos \frac{3 \delta_{12}^{\prime}}{2} \sin \frac{\delta_{12}^{\prime}}{2} \\
& \left(M_{\mathrm{T}_{12}} E_{\mathrm{T}_{12}^{1}}+r_{1}-r_{2}^{\prime}\right) \cos \frac{\delta_{12}^{\prime}}{2} \sin \frac{3 \delta_{12}^{\prime}}{2}=-M_{\mathrm{T}_{12}} E_{\mathrm{T}_{12}^{2}} \sin \frac{3 \delta_{12}^{\prime}}{2} \sin \frac{\delta_{12}^{\prime}}{2}
\end{aligned}
$$

Summing the obtained equations

$$
\left[\left(M_{\mathrm{T}_{12}} E_{\mathrm{T}_{12}^{1}}+r_{1}-r_{2}^{\prime}\right) \cos \frac{\delta_{12}^{\prime}}{2}+M_{\mathrm{T}_{12}} E_{\mathrm{T}_{12}^{2}} \sin \frac{\delta_{12}^{\prime}}{2}\right]\left(\cos \frac{3 \delta_{12}^{\prime}}{2}+\sin \frac{3 \delta_{12}^{\prime}}{2}\right)=0
$$

Therefore,

$$
\left(M_{\mathrm{T}_{12}} E_{\mathrm{T}_{12}^{1}}+r_{1}-r_{2}^{\prime}\right) \cos \frac{\delta_{12}^{\prime}}{2}+M_{\mathrm{T}_{12}} E_{\mathrm{T}_{12}^{2}} \sin \frac{\delta_{12}^{\prime}}{2}=0 \quad \text { or } \quad \cos \frac{3 \delta_{12}^{\prime}}{2}+\sin \frac{3 \delta_{12}^{\prime}}{2}=0
$$

Lemma V.2 If $\cos \frac{3 \delta_{12}^{\prime}}{2}+\sin \frac{3 \delta_{12}^{\prime}}{2}=0$, then there exists an $\mathcal{O}_{2}^{\prime}$ such that the reference circle $\mathcal{O}_{r}$ is a semicircle and $n=1$.

Proof Assume $\cos \frac{3 \delta_{12}^{\prime}}{2}+\sin \frac{3 \delta_{12}^{\prime}}{2}=0$. Then $\delta_{12}^{\prime}=\pi / 2$ and $r_{2}^{\prime}=r_{1}-\sqrt{2} r_{r}$ because $0<\delta_{12}^{\prime}<\pi$ from the definition of $\delta_{12}^{\prime}$. By the horizontal feasibility condition $9, \delta_{i f} \in\{n \pi \mid n \in \mathbb{N}\}$ if $2 n \delta_{12}^{\prime} \leq \pi$, and $\delta_{i f} \in$ $\{\pi-n \pi \mid n \in \mathbb{N}\}$ if $2 n \delta_{12}^{\prime}>\pi$. Since $0<\delta_{i f} \leq \pi$ and $n \in \mathbb{N}, \delta_{i f}=\pi$ and $n=1$ if $\mathrm{f} 2 n \delta_{12}^{\prime} \leq \pi$. Therefore, the reference circle is a semicircle, and $r_{r}=\left\|\vec{r}_{c_{1} c_{f}}\right\| / 2$. Then $r_{2}^{\prime}=r_{1}-\sqrt{2}\left\|\vec{r}_{c_{1} c_{f}}\right\| / 2$, and $2\left(r_{1}-r_{2}^{\prime}\right)=\sqrt{2}\left\|\vec{r}_{c_{1} c_{f}}\right\|>\left\|\vec{r}_{c_{1} c_{f}}\right\|$. By the existence and uniqueness theorem for convex cyclic polygons, ${ }^{14}$ such a cyclic polygon is unique. Therefore, $\exists$ a changed $\mathcal{O}_{2}^{\prime}$ such that the reference circle is a semicircle and $n=1$.

$$
\text { If } \begin{aligned}
&\left(M_{\mathrm{T}_{12}} E_{\mathrm{T}_{12}^{1}}+r_{1}-r_{2}^{\prime}\right) \cos \frac{\delta_{12}^{\prime}}{2}+ M_{\mathrm{T}_{12}} E_{\mathrm{T}_{12}^{2}} \sin \frac{\delta_{12}^{\prime}}{2}=0, \text { then } \\
& \tan \frac{\delta_{12}^{\prime}}{2}+\frac{E_{\mathrm{T}_{12}^{1}}}{E_{\mathrm{T}_{12}^{2}}}+\frac{2 r_{r}}{M_{\mathrm{T}_{12}} E_{\mathrm{T}_{12}^{2}}} \frac{\delta_{12}^{\prime}}{2}=0
\end{aligned}
$$

because $0<\delta_{12}^{\prime}<\pi$. Substituting the horizontal feasibility condition 9 into the above equation, we obtain the horizontal feasibility condition for the TDV with transition

$$
\begin{array}{r}
\tan \frac{\delta_{i f}}{4 n}+\frac{2 r_{r}}{M_{\mathrm{T}_{12}} E_{\mathrm{T}_{12}^{2}}} \sin \frac{\delta_{i f}}{4 n}+\frac{E_{\mathrm{T}_{12}^{1}}}{E_{\mathrm{T}_{12}^{2}}}=0 \quad \text { if } 2 n \delta_{12} \leq \pi \\
\tan \frac{\pi-\delta_{i f}}{4 n}+\frac{2 r_{r}}{M_{\mathrm{T}_{12}} E_{\mathrm{T}_{12}^{2}}} \sin \frac{\pi-\delta_{i f}}{4 n}+\frac{E_{\mathrm{T}_{12}^{1}}}{E_{\mathrm{T}_{12}^{2}}}=0 \quad \text { if } 2 n \delta_{12}>\pi
\end{array}
$$

Suppose $\psi_{\mathrm{T}_{21}}=5 \pi / 2+2 \delta_{12}^{\prime}$. Then $\psi_{1}=3 \pi / 2+\delta_{12}^{\prime}$. Following a similar process using the trigonometric identities,

$$
\left[\left(M_{\mathrm{T}_{12}} E_{\mathrm{T}_{12}^{1}}+r_{1}-r_{2}^{\prime}\right) \cos \delta_{12}^{\prime}-M_{\mathrm{T}_{12}} E_{\mathrm{T}_{12}^{2}} \sin \delta_{12}^{\prime}\right]\left(\cos 3 \delta_{12}^{\prime}+\sin 3 \delta_{12}^{\prime}\right)=0
$$


If $\left(M_{\mathrm{T}_{12}} E_{\mathrm{T}_{12}^{1}}+r_{1}-r_{2}^{\prime}\right) \cos \delta_{12}^{\prime}-M_{\mathrm{T}_{12}} E_{\mathrm{T}_{12}^{2}} \sin \delta_{12}^{\prime}=0$, then

$$
\tan \frac{\delta_{12}^{\prime}}{2}-\frac{E_{\mathrm{T}_{12}^{1}}}{E_{\mathrm{T}_{12}^{2}}}-\frac{2 r_{r}}{M_{\mathrm{T}_{12}} E_{\mathrm{T}_{12}^{2}}} \frac{\delta_{12}^{\prime}}{2}=0
$$

because $0<\delta_{12}^{\prime}<\pi$. Substituting the horizontal feasibility condition 9 into the above equation, we obtain the horizontal feasibility condition for the TDV with transition

$$
\begin{array}{r}
\tan \frac{\delta_{i f}}{4 n}-\frac{2 r_{r}}{M_{\mathrm{T}_{12}} E_{\mathrm{T}_{12}^{2}}} \sin \frac{\delta_{i f}}{4 n}-\frac{E_{\mathrm{T}_{12}^{1}}}{E_{\mathrm{T}_{12}^{2}}}=0 \quad \text { if } 2 n \delta_{12} \leq \pi \\
\tan \frac{\pi-\delta_{i f}}{4 n}-\frac{2 r_{r}}{M_{\mathrm{T}_{12}} E_{\mathrm{T}_{12}^{2}}} \sin \frac{\pi-\delta_{i f}}{4 n}-\frac{E_{\mathrm{T}_{12}^{1}}}{E_{\mathrm{T}_{12}^{2}}}=0 \quad \text { if } 2 n \delta_{12}>\pi
\end{array}
$$

Suppose $\psi_{\mathrm{T}_{21}}=7 \pi / 2+2 \delta_{12}^{\prime}$. Then $\psi_{1}=5 \pi / 2+\delta_{12}^{\prime}$. We obtain the same horizontal feasibility condition for the TDV with transition as defined in Eq. 46. If $\psi_{\mathrm{T}_{21}}=7 \pi / 2-2 \delta_{12}^{\prime}$, we obtain the same horizontal feasibility condition for the TDV with transition as specified in Eq. 49. In order to determine the radius of the reference circle $\mathcal{O}_{r}$, we determine the minimum number of alternate turning arcs in $\mathcal{O}_{r}$. In our previous work, ${ }^{5}$ the reference arc representing a straight line was used to compute the minimum number of alternate turning arcs with transition in $\mathcal{O}_{r}$. As $\lambda$ goes to $-\infty$ in Eq. 4, the arc $a_{r}$ goes to a straight line. Therefore, we use the number of alternating turning arcs referenced to the straight line connecting $c_{1}$ and $c_{f}$ as the minimum number $n_{m}$. With this direct (straight) reference line, the heading angles of the TDV with respect to $c_{1}$ at $s_{1}$ and $s_{\mathrm{T}_{21}}$ are given from Eqs. 29 to 32 by:

If $\operatorname{sgn}(\dot{\psi}) \geq 0$,

$$
\psi_{1}=\frac{3 \pi}{2} \quad \text { and } \quad \psi_{\mathrm{T}_{21}}=\frac{5 \pi}{2}
$$

If $\operatorname{sgn}(\dot{\psi})<0$

$$
\psi_{1}=\frac{\pi}{2} \quad \text { and } \quad \psi_{\mathrm{T}_{21}}=-\frac{\pi}{2}
$$

Also, $p_{4}$ in the transition arc $\mathrm{T}_{21}\left(M_{\mathrm{T}_{21}}, p_{3}, p_{4}\right)$ lies on the straight line. Then the $y$ component of $\mathcal{C}\left(s \mathrm{~T}_{21}\right)$ is equal to 0 . Using this information and following a similar procedure to obtain the horizontal feasibility condition for the TDV with transition,

$$
\mathcal{C}_{\mathrm{T}_{21}}=\left(2 M_{\mathrm{T}_{12}} E_{\mathrm{T}_{12}^{1}}+r_{1}\right) \hat{i}
$$

Note that we obtain the same $\mathcal{C}_{\mathrm{T}_{21}}$ regardless of the travel direction because of the straight line. In Theorem IV.1, the difference $r_{1}-r_{2}$ between two radii is important when we determine the minimum number of alternate turning arcs for the TDV without transition. Therefore, the difference $r_{1}-r_{2}^{\prime}$ in alternate turning arcs with transition is given by:

$$
r_{1}-r_{2}^{\prime}=M_{\mathrm{T}_{21}} E_{\mathrm{T}_{12}^{1}}
$$

Substituting into the equation of $n_{m}$ in Theorem IV.1, $n_{m}$ for the TDV with transition is given by:

$$
n_{m}=\left\{\begin{array}{cll}
\left\lceil\frac{\left\|\vec{r}_{c_{f}} c_{1}\right\|}{2 M_{\mathrm{T}_{21}} E_{\mathrm{T}_{12}^{1}}}\right\rceil & \text { if } & \frac{\left\|\vec{r}_{c_{f} c_{1}}\right\|}{2 M_{\mathrm{T}_{21}} E_{\mathrm{T}_{12}^{1}}} \notin \mathbb{N} \\
\left\lceil\frac{\left\|\vec{r}_{c_{f} c_{1}}\right\|}{2 M_{\mathrm{T}_{21}} E_{\mathrm{T}_{12}^{1}}}\right\rceil+1 & \text { if } & \frac{\left\|\vec{r}_{c_{f} c_{1}}\right\|}{2 M_{\mathrm{T}_{21}} E_{\mathrm{T}_{12}^{1}}} \in \mathbb{N}
\end{array}\right.
$$

\section{Example Landing Trajectories Possible with the TDV Solution}

A series of TDV solutions are presented in this section to illustrate the properties of a TDV solution over different distances, turning directions, and transition speeds. Unless otherwise indicated, the landing site is JFK Runway $31 \mathrm{~L}$ at latitude $40.6398^{\circ} \mathrm{N}$, longitude $73.7789^{\circ} \mathrm{W}$. For all cases we presume a minimum turn rate magnitude of $2.5 \mathrm{deg} / \mathrm{sec}$ and maximum turn rate magnitude of $7.5 \mathrm{deg} / \mathrm{sec}$ with a true airspeed of 225 $\mathrm{ft} / \mathrm{sec}$. Since this paper focuses on lateral plane TDV solutions, vertical flight path is not discussed. 
Figure 5 illustrates a series of TDV paths with different transition rates. The TDV initial state is latitude $40.69^{\circ} \mathrm{N}$, longitude $73.72^{\circ} \mathrm{W}$, heading $210^{\circ}$. In this case $r_{M}=5148.6315 \mathrm{ft}, r_{m}=1716.2105 \mathrm{ft}$, and $\left\|\vec{r}_{c_{1} c_{f}}\right\|=27076.9137 \mathrm{ft}$. Turning direction is clockwise (negative turn rate) as shown from an initial state $p_{0}$ to final state at JFK 31L. The leftmost subfigures, (a), (d), and (g), provide overhead views of the lateral TDV paths for the three subcases. The center subfigures show radius of curvature versus traversal distance $s$ over a single TDV sequence, illustrating rapid (b), moderate (e), and slow (h) transition rates. The rightmost subfigures show how heading changes for these three cases over single TDV sequence. Parameter values for cases (a)-(c), (d)-(f), (g)-(i), respectively, are given by: $M_{\mathrm{T}_{21}}=8,1.5,0.75, r_{r}=47518.64,15289.08,15283.68$ $\mathrm{ft}, r_{2}^{\prime}=1717.22,1829.61,2380.38 \mathrm{ft}$. As shown, the Figure (a) path minimally varies from the TDV path without transitions since the transitions are rapid. As the transitions slow, progress along the reference circle over each sequence diminishes, resulting in increased number of sequences from the minimum no-transition value of 4 sequences to 5 in subfigures (d)-(f) to 6 in subfigures (g)-(i).

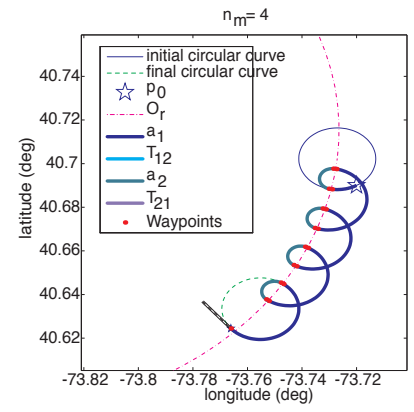

(a) $2 D$ TDV Trajectory for $M_{\mathrm{T}_{21}}=8$

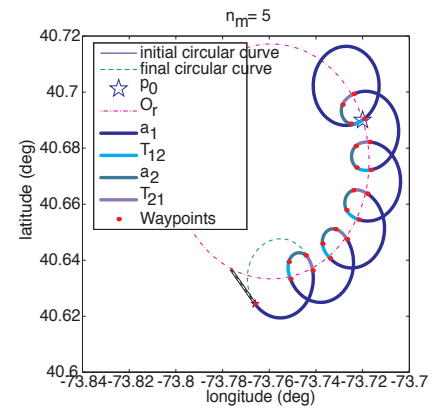

(d) $2 D$ TDV Trajectory for $M_{\mathrm{T}_{21}}=1.5$

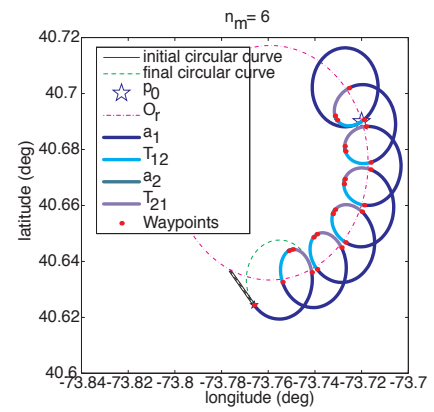

(g) $2 D$ TDV Trajectory for $M_{\mathrm{T}_{21}}=0.75$

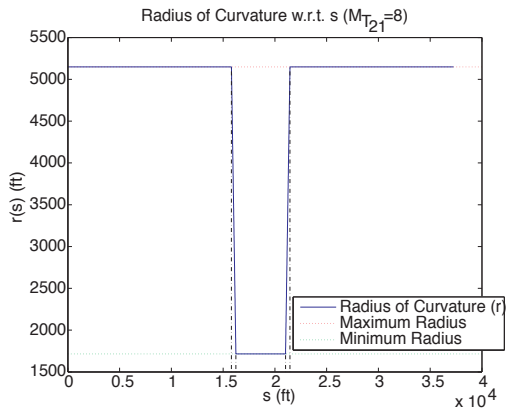

(b) Radius of Curvature for $M_{\mathrm{T}_{21}}=8$

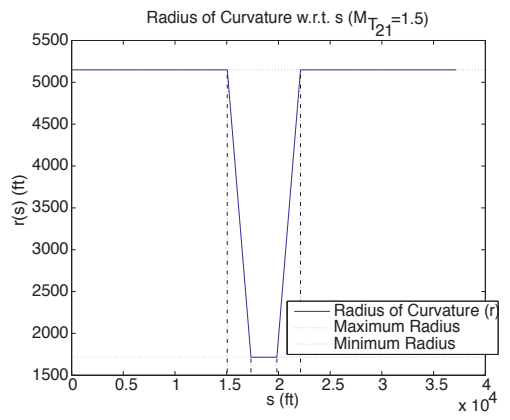

(e) Radius of Curvature for $M_{\mathrm{T}_{21}}=1.5$

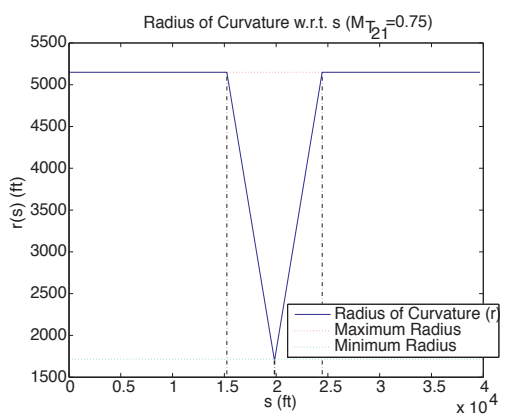

(h) Radius of Curvature for $M_{\mathrm{T}_{21}}=0.75$

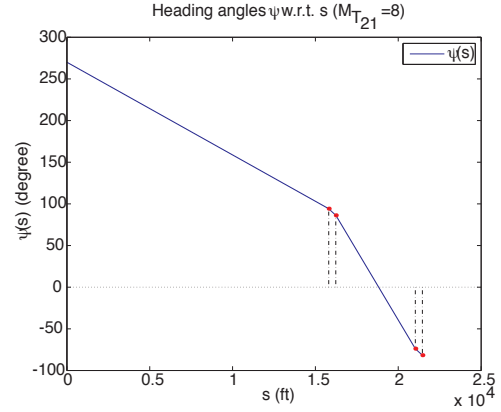

(c) Heading Angles for $M_{\mathrm{T}_{21}}=8$

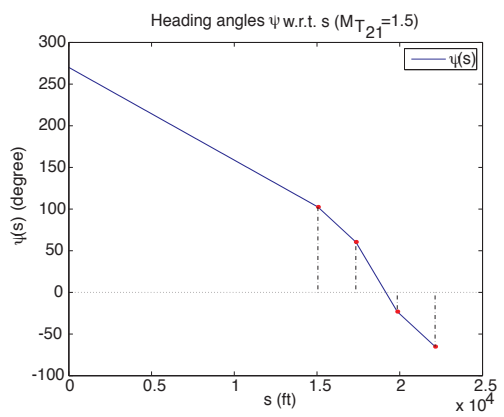

(f) Heading Angles for $M_{\mathrm{T}_{21}}=1.5$

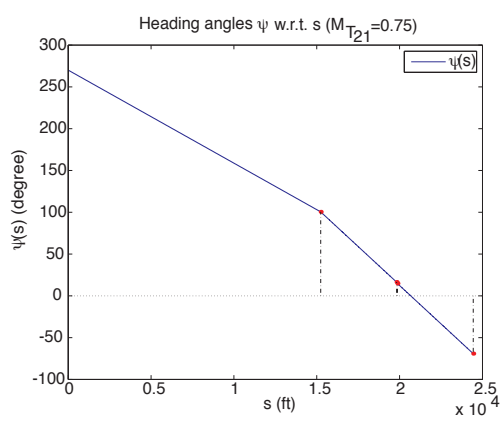

(i) Heading Angles for $M_{\mathrm{T}_{21}}=0.75$

Figure 5. TDV Trajectories to the JFK $31 L$

Figure 6 illustrates dual solutions over the two reference circle options available to the TDV solver for the same initial and final positions but different headings. In subfigure (a), the initial and final headings are the same as for the Figure 5 cases, with negative (clockwise) turn rate. For subfigure (b) the initial heading is the same (210) but the final landing site is JFK 13R rather than JFK 31L to provide a counterexample 
with positive (counterclockwise) turn rate. In both these cases, the distance between initial and final states is relatively large, resulting in multiple turning sequences $\left(n_{m}=5\right.$ for subfigure (a), $n_{m}=4$ for subfigure (b)).

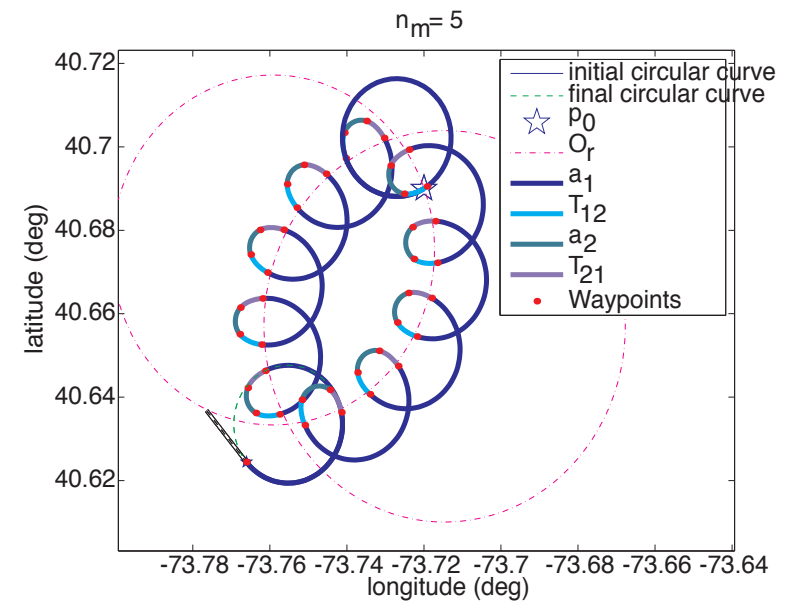

(a) $\operatorname{sgn}(\dot{\psi})<0$

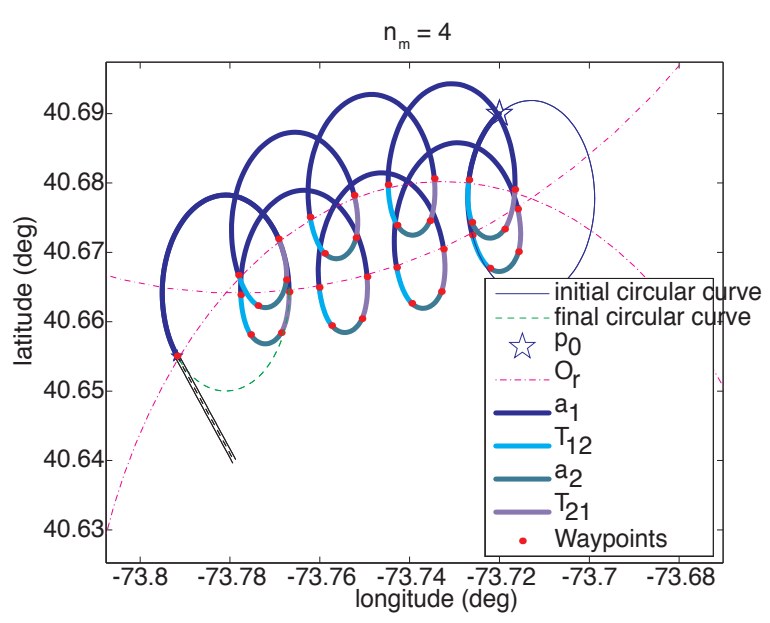

(b) $\operatorname{sgn}(\dot{\psi})>0$

Figure 6. TDV Trajectories to the JFK $31 L$ for $M_{\mathrm{T}_{21}}=1.5$

Figure 7 illustrates TDV solutions for situations in which the initial and final states are in close proximity relative to the TDV turning radii. In subfigure (a), travel direction is negative, and a single TDV sequence connects initial and final state. In subfigure (b), travel direction is positive, and the pair of solutions about the two possible reference circles requires two TDV turning sequences to reach JFK 31L. In both subfigures, the initial state has latitude $40.64^{\circ} \mathrm{N}$, longitude $73.77^{\circ} \mathrm{W}$, and heading $210^{\circ}$, with airspeed for this case set to 250 knots. These conditions result in $r_{M}=5722.3935 \mathrm{ft}, r_{m}=1907.4645$ $\mathrm{ft}$, and $\left\|\vec{r}_{c_{1} c_{f}}\right\|=9968.2462 \mathrm{ft}$. We presume transition rate $M_{\mathrm{T}_{21}}=1.5$ in this figure. $r_{r}$ is defined conditionally as $r_{r}=\left\{43740 \mathrm{ft}\right.$, sgn $\left(k_{c_{i} \times c_{f}}\right)<0 ; 79534 \mathrm{ft}$, $\left.\operatorname{sgn}\left(k_{c_{i} \times c_{f}}\right)>0\right\}$ for subfigure (a) and $r_{r}=$ $\left\{26214 \mathrm{ft}\right.$, sgn $\left(k_{c_{i} \times c_{f}}\right)<0 ; 10183 \mathrm{ft}$, sgn $\left.\left(k_{c_{i} \times c_{f}}\right)>0\right\}$ for subfigure (b). Similarly, $r_{2}^{\prime}$ is defined conditionally as $r_{2}^{\prime}=\left\{1363 f t\right.$, sgn $\left(k_{c_{i} \times c_{f}}\right)<0 ; 217.9 f t$, sgn $\left.\left(k_{c_{i} \times c_{f}}\right)>0\right\}$ for subfigure (a) and $r_{2}^{\prime}=\left\{2228 \mathrm{ft}\right.$, sgn $\left(k_{c_{i} \times c_{f}}\right)$ $\left.<0 ; 1946 f t, \operatorname{sgn}\left(k_{c_{i} \times c_{f}}\right)>0\right\}$ for subfigure (b).

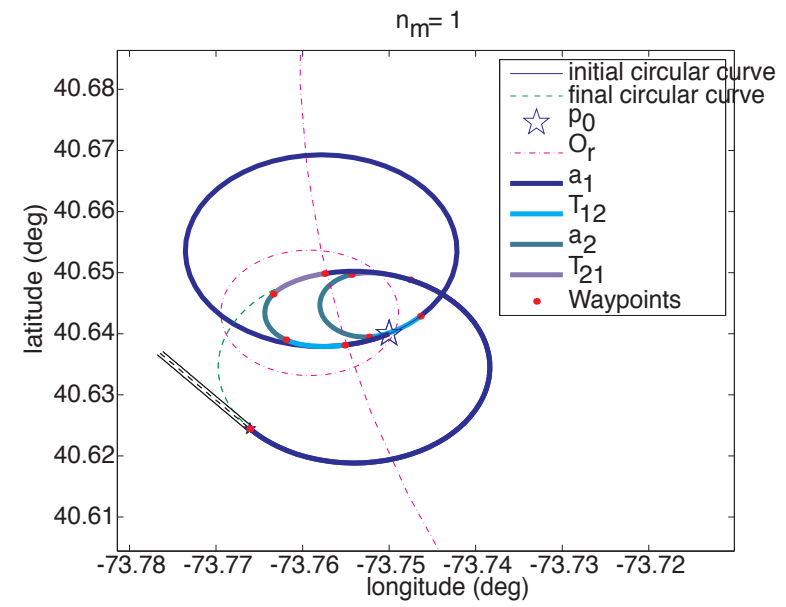

(a) $\operatorname{sgn}(\dot{\psi})<0$

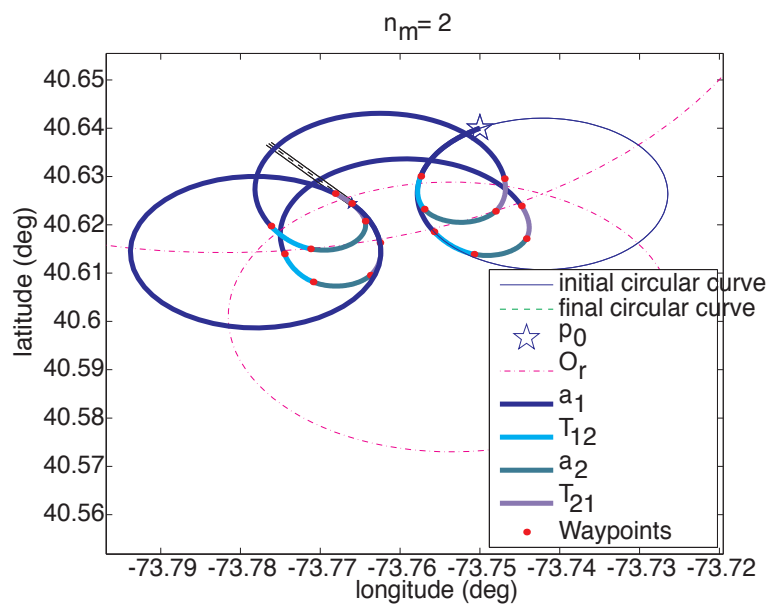

(b) $\operatorname{sgn}(\dot{\psi})>0$

Figure 7. TDV Trajectories to the JFK $31 L$ for $M_{\mathrm{T}_{21}}=1.5$ 


\section{Conclusions and Future Work}

This paper has presented an analytic trajectory planning method in which minimum and maximumradius turning flight segments are sequenced and connected with analytically-derived smooth transitions. A solution, described as a Turning Dubins Vehicle (TDV) path, is analytical in nature thus can be generated without substantial computational overhead. Given initial and final positions and headings, the TDV solver generates a reference circle that connects these states. We have shown the resulting solution exists and is unique over the comprehensive set of possible initial and final states in the lateral plane. A set of feasible landing trajectories comprised of alternating maximum and minimum radius turning radius segments can be used to guide an aircraft autonomously or used as a "geometric advisor" to the flight crew. To promote safety through a minimum-length but feasible solution, the minimum number of turning sequences for the TDV is determined, and we have shown that a path following the associated reference circle is feasible and has a minimum-length flight path. This paper extends a traditional Dubins path solver to cases in which straight flight is not possible, providing a computationally-efficient alternative to optimization or searchbased solvers. Emphasis in this paper is placed on definition of the TDV and analytically incorporating realistic transitions between minimum and maximum radius turning arcs.

In this paper, we have derived a 2-D TDV trajectory, i.e., a constant-altitude path. In future work, the sequence of alternate turning arcs must be extended to three-dimensional space that also manages airspeed and flight path angle to ensure longitudinal plane (and coupled) performance constraints are also satisfied. A steady descent flight path angle may be sufficient in some damage situations, although large-magnitude descents to the landing site will generally require extending the minimum-length TDV solution by inserting additional turning sequences. Flight performance envelope degradation may also require a more complex coupling between lateral and longitudinal path, as would be the case if flight path and airspeed constraints differ for minimum versus maximum radius turning arcs. We plan to focus on a full 3-D TDV implementation in near-term future work and incorporate this solver into a realistic flight management environment to enable evaluation of appropriate pilot interfaces as well as applicability to identified severe damage and failure scenarios.

\section{Acknowledgments}

The authors would like to thank collaborator Robert Sanner from the University of Maryland for his valuable insights and contributions to the adaptive flight planner. This research was supported in part under the NASA Aviation Safety program, Integrated Resilient Aircraft Control (IRAC) NRA Cooperative Agreement NNX08AB93A.

\section{References}

${ }^{1}$ Atkins, E., Portillo, A., and Strube, M., "Emergency Flight Planning applied to Total Loss of Thrust," Journal of Aircraft, Vol. 43, No. 4, Jul.-Aug. 2006, pp. 1205-1216.

${ }^{2}$ Strube, M., Sanner, R., and Atkins, E., "Dynamic Flight Guidance Recalibration after Actuator Failure," Proceedings of the 1st AIAA Intelligent Systems Technical Conference, Chicago, Illinois, Sep. 2004.

${ }^{3}$ Tang, Y., Atkins, E., and Sanner, R., "Emergency Flight Planning for a Generalized Transport Aircraft with Left Wing Damage," Proceedings of the AIAA Guidance, Navigation, and Control Conference, Hilton Head, South Carolina, Aug. 2007.

${ }^{4}$ Zhao, Y. and Tsiotras, P., "Mesh Refinement Using Density Function for Solving Optimal Control Problems," AIAA Infotech@Aerospace Conference, Seattle, Washington, Apr. 2009.

${ }^{5}$ Choi, H. and Atkins, E., "An Analytic Trajectory Planner for Aircraft with Severe Damage or Failures," Proceedings of the AIAA Infotech@Aerospace Conference, Seattle, Washington, Apr. 2009.

${ }^{6}$ Chen, T. and Pritchett, A., "Development and Evaluation of a Cockpit Decision-Aid for Emergency Trajectory Generation," Journal of Aircraft, Vol. 38, No. 5, Sep.-Oct. 2001, pp. 935-943.

${ }^{7}$ Saunders, J., Beard, R., and McLain, T., "Obstacle Avoidance Using Circular paths," Proceedings of the AIAA Guidance, Navigation, and Control Conference, Hilton Head, South Carolina, Aug. 2007.

${ }^{8}$ Brinkman, K. and Visser, H., "Optimal Turn-Back Maneuver after Engine Failure in a Single-Engine Aircraft during Climb-Out," Proceedings of the Institution of Mechanical Engineers - Part G: Journal of Aerospace Engineering, Vol. 221, No. 1, 2007, pp. 17-27.

${ }^{9}$ Boskovic, J., Prasanth, R., and Mehra, R., "A Multi-Layer Autonomous Intelligent Control Architecture for Unmanned Aerial Vehicles," AIAA Journal of Aerospace Computing, Information, and Communication, Vol. 1, Dec. 2004, pp. 605-628.

${ }^{10}$ Frazzoli, E., Dahleh, M. A., and Feron, E., "Maneuver-based motion planning for nonlinear systems with symmetries," IEEE Transactions on Robotics and Automation, Vol. 21, No. 6, Dec. 2005, pp. 1077-1091. 
${ }^{11}$ Ramsay, A. and Richtmyer, R. D., Introduction to Hyperbolic Geometry, Volume 1994, Sringer, 1995.

${ }_{12}$ Massey, W., A Basic Course in Algebraic Topology, Springer, 1997.

${ }^{13}$ Alexander, D. C. and Koeberlein, G. M., Elementary Geometry for College Students, Houghton Mifflin, 1999.

${ }^{14}$ Pinelis, I., "Cyclic polygons with given edge lengths: Existence and uniqueness," Journal of Geometry, Vol. 82, No. 1-2, Aug. 2005, pp. 156-171.

${ }^{15}$ Ross, K. A., Elementary Analysis: The Theory of Calculus, Springer Science \& Business, 8th ed., 2003.

${ }^{16}$ Boyd, S. and Vandenberghe, L., Convex Optimization, Cambridge University Press, 2004.

${ }^{17}$ Prástaro, A. and Rassias, T. M., Geometry in Partial Differential Equations, World Scientific, 1994. 